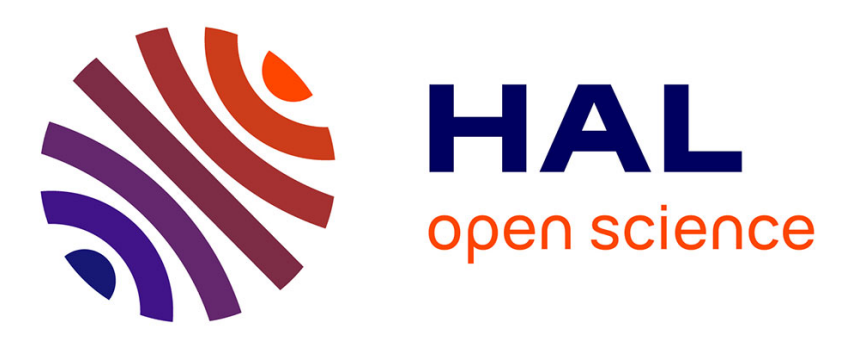

\title{
Bioinspired multilayered cellular composites with enhanced energy absorption and shape recovery
}

Huan Jiang, Louise Le Barbenchon, Brett A Bednarcyk, Fabrizio Scarpa, Yanyu Chen

\section{- To cite this version:}

Huan Jiang, Louise Le Barbenchon, Brett A Bednarcyk, Fabrizio Scarpa, Yanyu Chen. Bioinspired multilayered cellular composites with enhanced energy absorption and shape recovery. Additive Manufacturing, 2020, 36, pp.101430. 10.1016/j.addma.2020.101430 . hal-03472100

\section{HAL Id: hal-03472100 https://hal.science/hal-03472100}

Submitted on 9 Dec 2021

HAL is a multi-disciplinary open access archive for the deposit and dissemination of scientific research documents, whether they are published or not. The documents may come from teaching and research institutions in France or abroad, or from public or private research centers.
L'archive ouverte pluridisciplinaire HAL, est destinée au dépôt et à la diffusion de documents scientifiques de niveau recherche, publiés ou non, émanant des établissements d'enseignement et de recherche français ou étrangers, des laboratoires publics ou privés. 


\title{
Bioinspired multilayered cellular composites with enhanced energy absorption and
}

\section{shape recovery}

Huan Jiang ${ }^{\mathrm{a}}$, Louise Le Barbenchon ${ }^{\mathrm{b}}$, Brett A. Bednarcyk ${ }^{\mathrm{c}}$, Fabrizio Scarpa ${ }^{\mathrm{d}}$, Yanyu Chen ${ }^{\mathrm{a} *}$

${ }^{a}$ Department of Mechanical Engineering, University of Louisville, Louisville, KY 40292, USA

${ }^{\mathrm{b}}$ Arts et Métiers ParisTech, CNRS, I2M Bordeaux, Esplanade des Arts et Métiers, Talence Cedex

$$
\text { F-33405, France }
$$

${ }^{\mathrm{c}}$ NASA Glenn Research Center, Cleveland, $\mathrm{OH} 44135$, USA

${ }^{\mathrm{d}}$ Bristol Composites Institute (ACCIS) and Dynamics and Control Research Group (DCRG),

University of Bristol, BS8 1TR Bristol, UK

*Corresponding Author, E-mail: Yanyu.chen@ louisville.edu

\begin{abstract}
Inspired by the multiscale configuration of the microstructure of cork, the paper describes the design, 3D printing, and evaluation of a new type of multilayered cellular composite (MCC) structure composed of hard brittle and soft flexible phases. The mechanical behavior of 3D printed MCC structures have been investigated both experimentally and numerically. The experiments show that the MCC structure absorbs four times the amount of energy of a conventional cellular configuration under compressive strains up to $70 \%$. Finite element simulations and 2D digital image correlation (DIC) also show that the multilayered architecture provides a more uniform strain distribution and higher stress transfer efficiency, with a resulting progressive failure mode rather than a catastrophic one. Cyclic loading tests demonstrate that the MCC structure also possesses exceptional shape recoverability under compressive deformations up to $40 \%$. These remarkable performance characteristics result from synergies between the properties of the two constituent materials and the chosen multilayered cellular microstructure. The soft phase, in particular, plays a pivotal role in absorbing elastic
\end{abstract}


energy during loading and then releasing the stored energy while unloading. The volume fraction of the soft phase is also essential to control energy absorption and the transition of failure modes. The deformation mechanisms demonstrated here are robust and applicable to other architected cellular materials across multiple length scales and suggest new ways to design lightweight and high-resilience structural materials.

Keywords: bioinspired composites, cellular structures, 3D printing, energy absorption, shape recovery

\section{Introduction}

Advanced structural materials featuring a combination of lightweight, exceptional stiffness, strength, energy absorption, and damage tolerance are becoming crucially important for multifunctional applications in aerospace, biomedical, semiconductor, and automotive engineering. Lightweight structural materials in automotive engineering offer significant potential to increase vehicle safety and efficiency [1]. A $10 \%$ reduction in vehicle weight can result in a 6\%-8\% fuel economy improvement [2]. This is particularly important for hybrid, plugin hybrid and electric vehicles, for which lightweight materials improve operational efficiency and increase their all-electric range [3,4]. Mechanical energy absorption and structural integrity are also essential for automotive structural components to function properly [5-8]; vehicle components such as door assemblies and bumper beams must be robust enough to maintain the safety of passengers even for severe crash conditions $[9,10]$. Lightweight, cost-efficient materials and structures are also critical to next-generation aerospace vehicles, particularly emerging electric and hybrid-electric aircraft. To this end, intensive efforts have been devoted to developing lightweight structural composites with exceptional stiffness, strength, impact resistance, and durability. Despite recent advances in materials and manufacturing techniques, 
the design and fabrication of low-cost structural materials with lightweight and superior mechanical performance still represent a challenge.

Biological structural materials such as nacre, glass sea sponges, and cellular solids feature unusual mechanical properties that are difficult to reproduce in man-made and artificial materials. For example, the hierarchical assembly of brittle minerals and soft proteins provides nacreous composites an unusual combination of stiffness, strength, damage tolerance, and impact resistance [11-14]. Recent advances in additive manufacturing such as multi-material and multiscale 3D printing have, however, enabled the production of bioinspired nacreous composites by emulating internal architectures and compositions [15-18]. Similar microstructure designs with the synergistic interplay between hard brittle and soft organic layers have also been identified in glass sea sponges [19-22], bones [23,24], and teeth [25,26]. Cellular solids represent another type of lightweight structural system that can be found in biological materials such as wood and cork [27,28]. By engineering the topology of cellular materials, one can have lightweight, high specific strength, and energy absorption characteristics [29-33]. For example, sheet-based and strut-based gyroid cellular structures with graded density design were investigated. It is shown that graded cellular structures have better energy absorption than uniform ones [34]. Cellular auxetic structures subjected to quasi-static and dynamic loadings were studied both numerically and experimentally [35]. It was found that auxetic structures are superior to non-auxetic structures in terms of impact resistance and energy absorption. By using 3D printing techniques researchers have recently developed hierarchical honeycomb structures at cell-wall and macro cellular-structure levels with superior mechanical performance compared with their natural counterparts [36-38]. These numerical and experimental investigations indicate that natural materials are a rich source of inspiration for design principles to develop next- 
generation structural artificial solids. Most of the current efforts are still nevertheless focused on understanding the fundamental mechanical properties of bioinspired structural materials, such as stiffness, strength, and fracture toughness. Studies related to the mechanical behavior and failure modes under large deformations are somewhat limited, leaving new deformation mechanisms and optimal materials design principles untapped under such conditions.

This paper explores the quasi-static mechanical performance of bioinspired cellular composites under large deformation using a combined experimental and numerical approach. The material system investigated here is inspired by the multiscale nature of cork microstructure. Cork is originated from the bark of the oak tree Quercus Suber that grows in sub-desertic areas [39]. The microstructure of cork was initially investigated by Robert Hooke in 1664 using an optical microscope [40] and, in modern times, studied in detail by Gibson et al [39]. with a scanning electron microscope (SEM). Cork cells present a hexagonal prism shape (Figure 1 (a)) [41]. Due to this unique structure, cork exhibits some outstanding properties, in particular, superior energy absorption that can be used for impact-loaded applications [42]. Another distinctive characteristic of cork is the composition of its cell walls, involving several distinct material layers (Figure 1 (b)) [43-45]. The cork cell walls consist of a thin and stiff, lignin-rich middle lamella (internal primary wall), a thick secondary wall made up of alternating suberin and a wax lamella, and a final thin tertiary wall of polysaccharides (cellulose and hemicellulose). The exact chemical constitution depends, however, on factors such as the geographic origin, the climate and soil conditions, the genetic origin, the dimensions of the tree, together with its age and growth conditions [46,47]. From a mechanical viewpoint, the combination of its cellular structure and the specific chemical and geometric layout of the lamellae gives rise to the distinctive properties of cork. 
Inspired by these multiscale structural and material features observed in cork, a range of 3D MCC structures have been manufactured at the millimeter scale using a PolyJet multi-material 3D printer. Quasi-static mechanical testing and 2D digital image correlation (DIC) characterizations have been performed to identify the deformation mechanisms of the $3 \mathrm{D}$ printed MCC structures under large deformations. The proposed MCC structures show significant energy absorption, a beneficial progressive failure mode, and a remarkable shape recovery capability. FE simulations in combination with a user-defined constitutive model have been conducted to better understand the deformation mechanisms under large deformations. It is demonstrated that the design principles derived from the cork cell wall are transferable to manmade materials, and they can be employed to develop lightweight structural composites with enhanced mechanical performance.

\section{Materials and methods}

\subsection{Model design and multi-material $3 D$ printing}

The proposed multilayered cellular composite with a triply periodic minimal surface (TMPS) is inspired by the multi- material compositions of corks. TPMS models are mathematical surfaces that exhibit zero mean curvature. The smoothness of the surfaces and the absence of intersections enable the prevention of stress concentration $[48,49]$. Schwarz P minimal surface is one of the simplest TPMS models. For simplicity, the Schwarz P surface is chosen as our composite design. The level set function for the surface can be expressed as [50]

$$
f(x, y, z)=\cos (x)+\cos (y)+\cos (z)-\frac{1}{2} \cos (x) \cos (y)-\frac{1}{2} \cos (y) \cos (z)-\frac{1}{2} \cos (z) \cos (z)+t
$$

where the value of $t$ determines the volume fraction of inside and outside the surface. It is found that the minimal surface divides the repeating unit cells (RUCs) in an even manner when 
$t=-0.3095$ (Figure S1 (a)). Note that the lattice constant here is $a=2 \pi$ due to the $2 \pi$ periodicity of this minimal surface function. The lattice constant is scaled to $25 \mathrm{~mm}$ and the volume fraction of the constitutive solids is set as $25 \%$ because of the resolution of the $3 \mathrm{D}$ printer used here. VeroWhitePlus (RGD835) and Agilus 30 black are chosen as hard and soft phases, respectively. Homogeneous VeroWhitePlus (VW) structures are created by offsetting the minimal surface $1.318 \mathrm{~mm}$ both inward and outward (Figure S1 (b)). Similarly, the MCC structure is constructed by offsetting the minimal surface with corresponding distances according to the volume fraction of each phase. Figure S1 (c) shows the MCC configuration with a total volume fraction of $25 \%$ for solids, where the volume fraction of the soft phase is $20 \%$. The geometric parameters for the MCC structures with different volume fractions are listed in Table S1. Furthermore, we have designed 3-layer, 5-layer, and 7-layer MCCs with VeroWhitePlus and Agilus 30. Due to the resolution of our printer, the 7-layer model has considerable defects. For 3layer composite, the hard phase is only constrained on one side, while it is constrained on both sides for 5-layer MCC. Therefore, the 5-layer design is chosen for our research. 
(a)

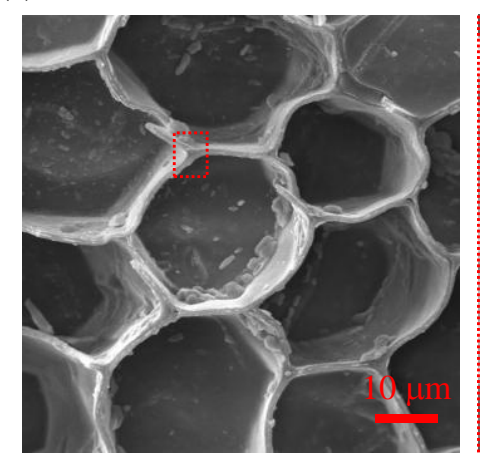

(e)

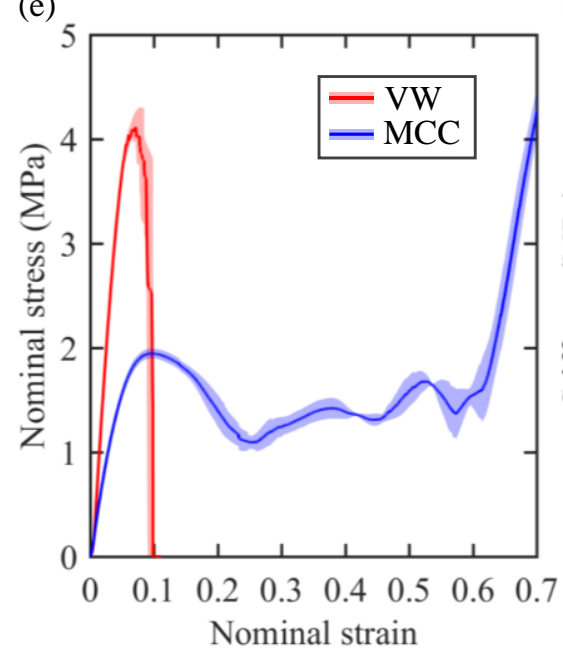

(b)

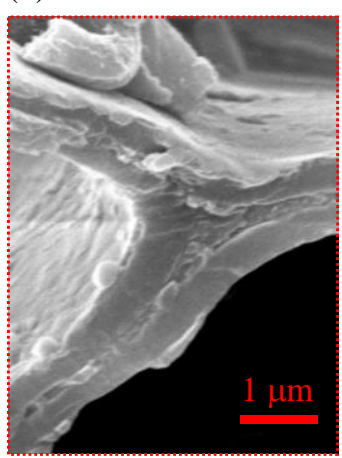

(f)

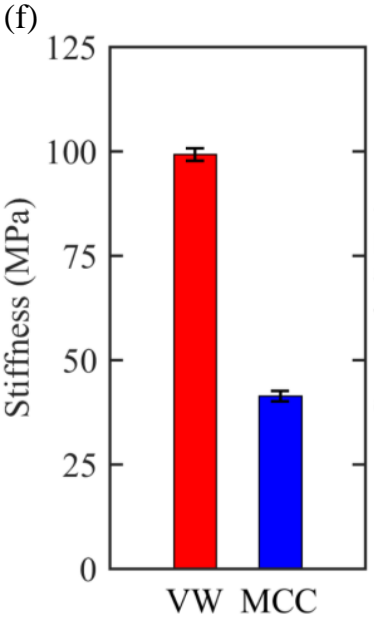

(c)

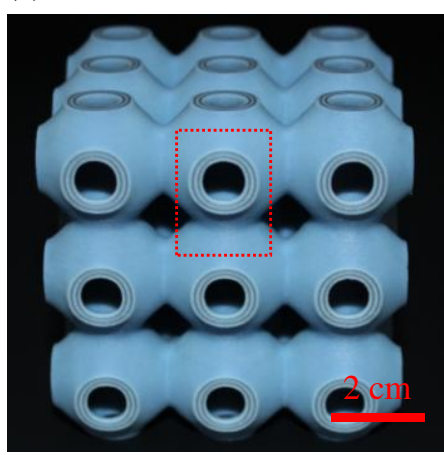

(g)

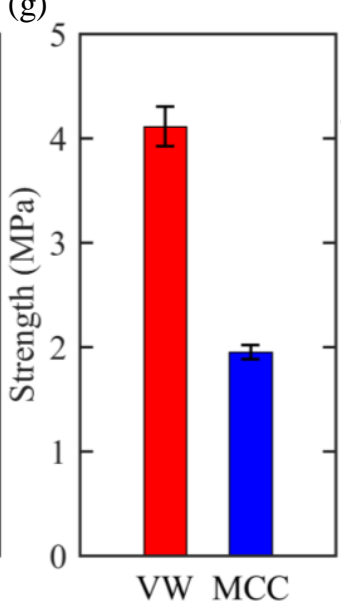

(d)

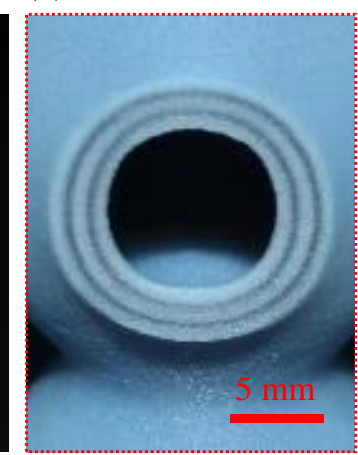

(h)

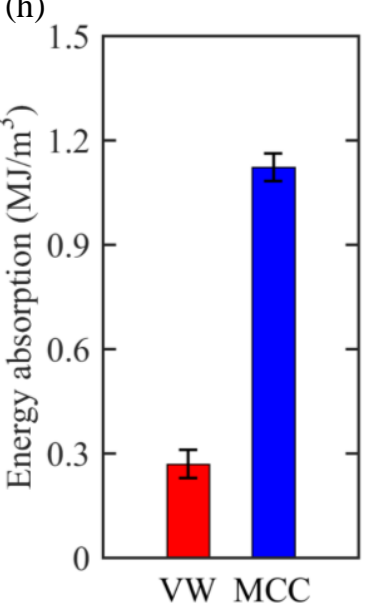

Figure 1. Bioinspired multilayered cellular composite design and uniaxial compression tests. (a)-

(b) Scanning electron microscope image of cork microstructure and its multilayered cell wall. (c)-(d) 3D printed cellular composite composed of five layers of hard and soft phases. The cellular structure is defined by a Schwarz P minimal surface. (e) Measured compressive stressstrain curves for the 3D printed MCC structure and a VW structure. (f)-(h) Comparisons between the measured stiffness, strength, and energy absorption, respectively.

Once the 3D printable geometric models are designed, we can fabricate the samples by using a multi-material PolyJet printer (Objet 260 Connex3, Stratasys, USA) with a resolution of $16 \mu \mathrm{m}$ . The photopolymer resin is ejected from the orifices of the print heads to the tray or the previous layer and then quickly cured with UV light [51]. Meanwhile, different photopolymer resins can 
be jetted together to tune the overall mechanical properties. After UV light cures one layer, the following layers are printed in the same way.

In the multilayered cellular shell, a brittle material (VeroWhitePlus (RGD835)) is chosen as the hard phase, while the employed flexible soft material is Agilus 30. Figure 2 shows the mechanical behavior of the VeroWhitePlus and the Agilus 30 from uniaxial tension tests under ASTM standard D638 and D412, respectively [52,53]. VeroWhitePlus yields at a 5\% strain and abruptly fails around $16 \%$ of tensile strain. The Agilus 30 polymer, on the other hand, shows a rubber-like hyperelastic behavior and provides remarkable elasticity and shape recovery. Clearly, VeroWhitePlus is much more brittle than Agilus 30. The printed MCC structure is composed of $3 \times 3 \times 3$ RUCs (Figures 1 (c)-(d)). The lattice constant of the RUC is $25 \mathrm{~mm}$ and the overall filled volume is $25 \%$. For each MCC structure, unless otherwise specified, the volume fractions of the VeroWhitePlus and the Agilus30 in the MCC structure are $80 \%$ and $20 \%$, respectively. For benchmark we have also printed the same cellular structure composed by VeroWhitePlus only; this configuration is denoted as the VW structure. Additional images for VW and MCC structures are shown in Figures S1 (d) and (e), respectively. 


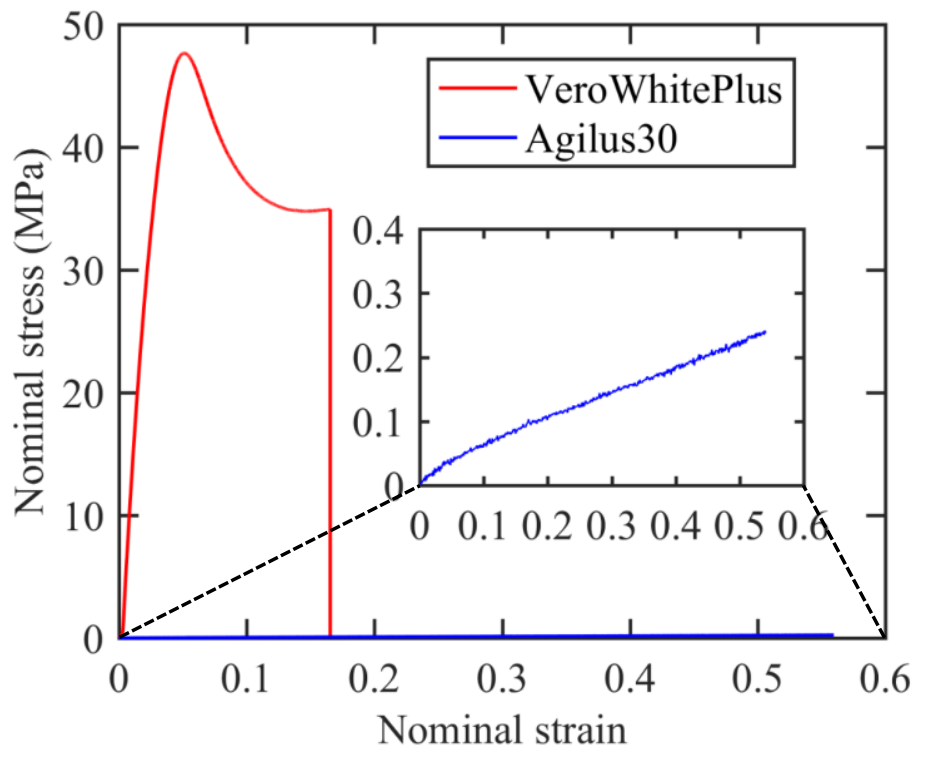

Figure 2. Mechanical properties of the VeroWhitePlus and Agilus 30 from uniaxial tensile tests on dogbone samples. Dimensions of each dogbone can be found in the corresponding ASTM standard.

\subsection{Mechanical testing of $3 D$ printed samples}

3D printed samples have been tested under displacement control in quasi-static uniaxial compression (Instron 5569A, US A) with a load cell of $50 \mathrm{KN}$. All samples were compressed to a maximum strain of $70 \%$. The Bluehill 2.0 software was connected to the Instron system to control the machine and acquire the data. Two circular smooth platens were used to transfer the compressive force from the load cell. The platens were cleaned with alcohol before each test. The cross-bar head moved at a speed of $2.5 \mathrm{~mm} / \mathrm{min}$, corresponding to a strain rate of $5.5 \times 10^{-4} \mathrm{~s}^{-1}$. Cyclic loading-unloading tests were again conducted through the Bluehill software by using a triangular waveform. Five cycles have been performed in total, with the most important parameters for these cyclic tests being the maximum and minimum displacements. The 
maximum corresponded to displacements related to $20 \%$ and $40 \%$ of compressive strain, while the minimum was set to 0 (i.e., the original position).

\subsection{Finite element simulations}

Modeling composite materials with multiple length scales is challenging when mesh is attempted at each scale. Advanced modeling frameworks such as multiscale modeling provide a powerful way to predict the mechanical performance of composite materials, especially when damage and fracture are involved [54-56]. In addition, when combined with topology optimization and machine learning, these efficient modeling frameworks can be employed to optimize the mechanical performance and functionalities of composites [57-59]. The purpose of the numerical simulations performed in this work is to provide additional information to reveal the deformation mechanisms. Therefore, conventional finite element simulations are implemented to understand the macroscopic mechanical behavior of MCCs. The mechanical behavior of the 3D printed samples was simulated using the finite element method (Abaqus 6.14, Dassault Systems, France). The geometric models of the VW and MCC structures were meshed using pre-processing software (Hypermesh, Altair Engineering Inc, USA) with the C3D8 element. Both the VW and the MCC structures were meshed using solid mapping in Hypermesh with 283392 elements. We have done convergence tests on FE models with 283392 elements and 198720 elements. The results indicate that the number of elements used in this work is sufficient to capture the mechanical response of the proposed architectures. The bottom boundary was constrained along the vertical direction, and a vertical displacement was applied to the top of the model. General self-contact interaction with a friction coefficient of 0.2 was defined for tangential motion and hard contact for the normal one. For the weak form of the dynamic 
equilibrium equation, one is encouraged to refer to references [60,61]. A dynamic explicit solver with an adaptive time-stepping was used to perform the simulations.

A user-defined viscoplastic constitutive model was developed for the VeroWhitePlus and embedded to ABAQUS through the VUMAT interface. The VUMAT code is available in the appendix of reference [62]. Tensile experiments were performed in different strain rates [62], i.e. $0.001 s^{-1}, 0.01 s^{-1}, 0.1 s^{-1}$, and a significant difference was reported. Therefore, it's necessary to choose a viscoplastic model to perform the simulation. This constitutive relation was initially developed for polymers $[63,64]$ and then widely used for 3D printed photopolymers $[62,65]$ such as VeroWhitePlus used in this work. The theoretical part of this constitutive model is described in reference [65], where backward Euler algorithm is used to update stress, strain, and state variables. For the detailed implementation of this VUMAT, one is recommended to refer to reference [65]. Due to the layer-by-layer feature and the imperfection involved during printing, the parameters of the VUMAT model will vary in a certain range. In this sense, uncertainty analysis will be essential to reveal their effect on the numerical results [66,67]. However, as a supplement way for the experiment, our FE analysis is applied to uncover the deformation mechanisms.

In this viscoplastic constitutive relation, the strain energy density for a hyperelastic spring is expressed as [68]:

$$
W=\mu\left\{\frac{1}{2}\left(\bar{I}_{1}-3\right)+\frac{1}{20 \lambda_{L}^{2}}\left(\bar{I}_{1}^{2}-9\right)+\frac{11}{1050 \lambda_{L}^{4}}\left(\bar{I}_{1}^{3}-27\right)+\frac{19}{7000 \lambda_{L}^{6}}\left(\bar{I}_{1}^{4}-81\right)+\frac{519}{673750 \lambda_{L}^{8}}\left(\bar{I}_{1}^{5}-243\right)+\frac{K_{0}}{2}\left(\frac{J_{e}^{2}-1}{2}-\ln J_{e}\right)\right\}
$$


where ${ }^{\mu}$ is the initial shear modulus, $\lambda_{L}$ is the limiting network stretch, $K_{0}$ is the initial bulk modulus, ${ }^{J}$ is the elastic volume ratio related to temperature. The first deviatoric strain invariant $\bar{I}_{1}$ is defined as follows [68]

$$
\begin{gathered}
\bar{I}_{1}=I_{1} J^{-2 / 3} \\
I_{1}=\operatorname{trace}(\boldsymbol{B})=B_{11}+B_{22}+B_{33}
\end{gathered}
$$

where $\boldsymbol{B}$ is the left Cauchy-Green deformation tensor. The total volumetric ratio $J$ is [68]

$$
J=\sqrt{\operatorname{det}(\boldsymbol{B})}
$$

When thermal effects are neglected, $J_{e}=J$.

For a viscoplastic element, the effective shear strain rate is determined by [62]

$$
\dot{\gamma}^{p}=\gamma_{0} \exp \left[-\frac{\Delta G}{\kappa \Theta}\left(1-\left(\frac{\sigma_{e}}{s}\right)^{5 / 6}\right)\right]
$$

where $\gamma_{0}$ is pre-exponential shear strain rate, $s$ is the thermal shear yield strength, $\kappa$ is the Boltzmann's constant, ${ }^{\sigma_{e}}$ is the effective stress, $\Delta G_{\text {is }}$ the initial free energy change.

The strain-softening in the viscoplastic element is determined through [62]

$$
\dot{s}=h\left(1-\frac{s}{s_{s}}\right) \dot{\gamma}^{p}
$$


where $\dot{s}$ is the rate of a thermal shear yield strength, $s_{s}$ is $s$ at the steady-state, $h_{\text {is }}$ the slope of softening part. The parameters used in this user-defined constitutive law are summarized in Table 1. Young's modulus is taken from the dogbone experiments, while Poisson's ratio is taken as the value that is widely used among researchers $[38,69]$. The other parameters are not universal, but they are adequate to capture the mechanical response of VeroWhitePlus under large deformation.

For the soft phase, an Arruda-Boyce hyperelastic model was used [70]. The parameters for $\mu, \lambda_{L}$, and $D$ are listed in Table 2 . Here, $D$ is material constant, and

$$
K_{0}=\frac{2}{D}
$$

Table 1. The parameters used for VeroWhitePlus in VUMAT

\begin{tabular}{cc}
\hline Material parameters & Values \\
\hline$E(\mathrm{MPa})$ & 1600 \\
$v$ & 0.33 \\
$\gamma_{0}$ & $5.0 \times 10^{5}$ \\
$\Delta G_{(J)}$ & $1.25 \times 10^{-19}$ \\
$s_{(\mathrm{MPa})}$ & 175 \\
$s_{s}(\mathrm{MPa})$ & 80 \\
$h_{(\mathrm{MPa})}$ & 500 \\
$\mu(\mathrm{MPa})$ & 4.5 \\
$\lambda_{L}$ & 3.5 \\
$K_{0}(\mathrm{MPa})$ & 15 \\
\hline
\end{tabular}

Table 2. Parameters for Arruda-Boyce hyperelastic model

\begin{tabular}{cc}
\hline Material parameters & value \\
\hline$\mu(\mathrm{MPa})$ & 0.18 \\
$\lambda_{L}$ & 2.05 \\
$D\left(\mathrm{MPa}^{-1}\right)$ & 0.002 \\
\hline
\end{tabular}




\section{Results and discussion}

\subsection{Uniaxial compression tests}

To understand the mechanical behavior of the MCC structure under large deformations, uniaxial compression tests with a strain rate of $5.5 \times 10^{-4} \mathrm{~s}^{-1}$ have been performed. Figure 1 (e) shows the experimental stress-strain curves for the VW and MCC structures up to a $70 \%$ compressive strain. Tests were repeated three times and we have observed good repeatability of the results from each test, especially within the small strain range, as indicated by the small variation of stress ranges in the shadow. For the VW structure, the stress increases rapidly until the strain reaches $7 \%$ and then drops abruptly to zero, associated with a catastrophic failure mode. The MCC structure is more compliant than the VW configuration, and the initial stress peak occurs at $10 \%$ strain, followed by a long stress plateau until approximately $60 \%$ of compression before densification. This stark contrast between the two layouts is due to the different failure modes for the VW and MCC structures, which will be discussed below. Comparisons of stiffness, strength and energy absorption between the VW and MCC configurations are summarized in Figures 1 (f)-(h). Here, energy absorption is calculated as the area under the stress-strain curve. The MCC shows a decrease in the stiffness and strength compared to the VW decreased by $58.3 \%$ and $52.6 \%$, respectively. The energy absorption is however increased by $317 \%$ in the case of the MCC configuration.

To explain the significant difference in the compressive stress-strain behavior, the measured mechanical deformation patterns of the VW and MCC structures are shown at different strain levels. As shown in Figure 3 (a), the VW structure begins to fracture locally at the bottom layer when the strain reaches $5.7 \%$ (Figure 3 (b) $\boldsymbol{A}$ ). Failure initiates in the bottom layer and locally 
within the top layer when the strain increases to 6.3\% (Figures 3 (b) $\boldsymbol{B}$ and $\boldsymbol{C}$ ). With the further release of strain energy, the fracture propagates, and the failure zone enlarges considerably when the compressive strain reaches $7.7 \%$ (Figure 3 (b) $\boldsymbol{D}$ ), followed by the structure breaking abruptly at $8.7 \%$ of strain (Figure 3 (b) $\boldsymbol{E}$ ). This deformation pattern indicates that the VW structure is relatively brittle, and it is not able to sustain large deformations characteristic of high energy-absorbing materials. Figure 3 (c) shows the deformation evolution of the MCC structure. The MCC configuration also fractures locally, however, the compressive strain has reached $10 \%$ by the time cracking is evident (Figure 3 (d) $\boldsymbol{F}$ ). The failure then propagates and expands to the whole MCC structure. It is worth noticing that most of the side holes fail at $14 \%$ of compressive strain (Figures 3 (d) $\boldsymbol{G}$ and $\boldsymbol{H}$ ). As the deformation progresses the structure begins to buckle at the bottom layer and engages contact between different layers. The middle layer fails at a global compressive strain of $28 \%$ (Figure 3 (d) I). With further increase of the compressive strain, the cell walls in the MCC structure completely collapse and contact with each other, giving a rapid increase in stress at a compressive strain of 58\% and gradually densifies towards the end (Figure 3 (d) $J$ ). Compared with the VW structure, the MCC configuration features a more stable deformation pattern and exhibits a combination of ductile-like and brittle-like behavior under large deformations. The significantly different deformation patterns observed during the mechanical tests qualitatively explain the enhanced energy absorption of the MCC configuration. 


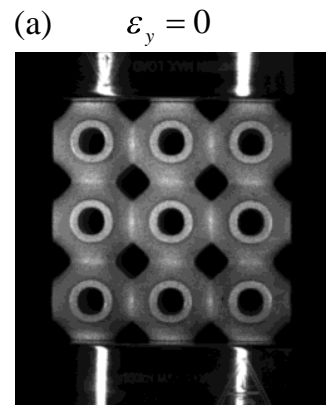

(b)

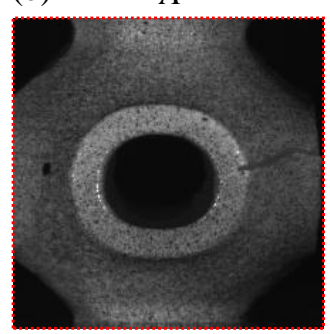

(c)

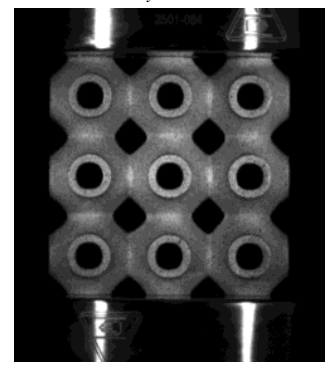

(d)

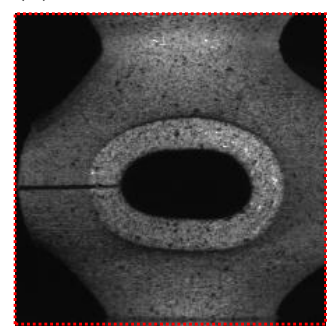

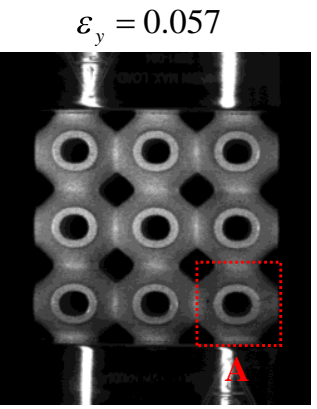

B

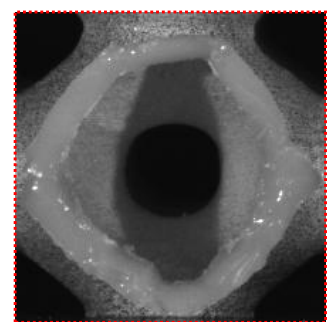

$\varepsilon_{y}=0.1$

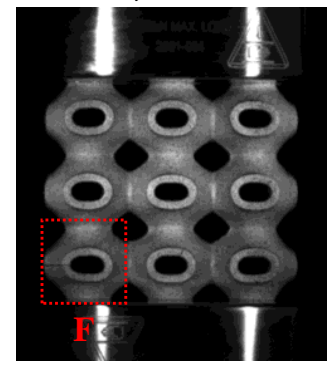

G

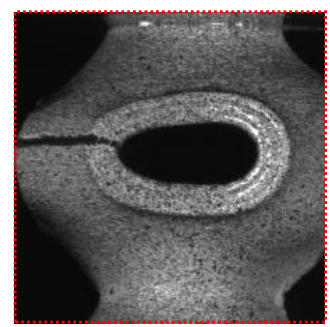

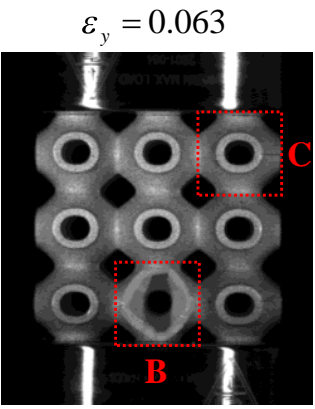

C

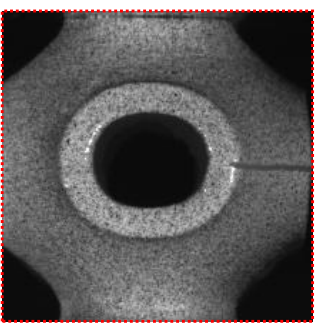

$\varepsilon_{y}=0.14$

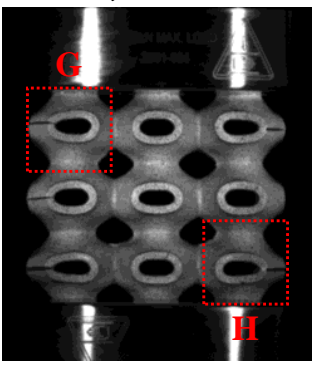

H

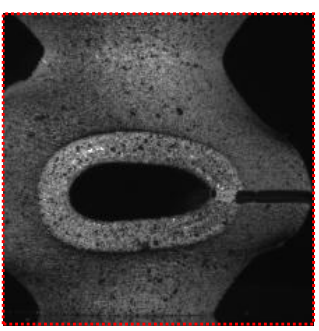

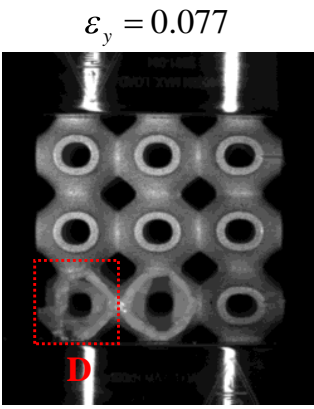

D

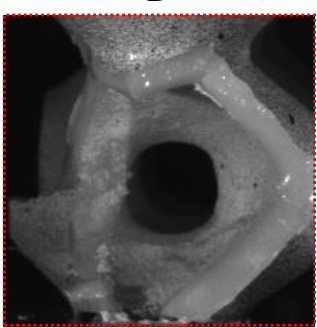

$\varepsilon_{y}=0.28$

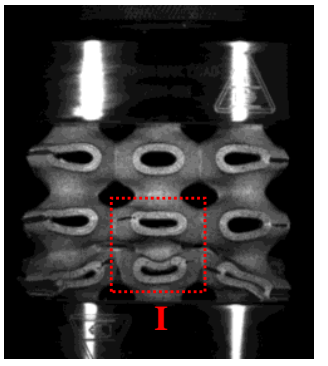

I

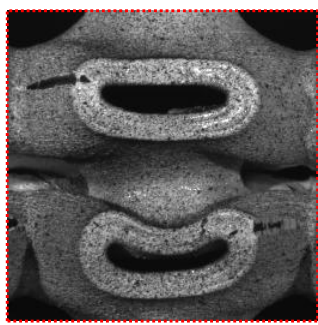

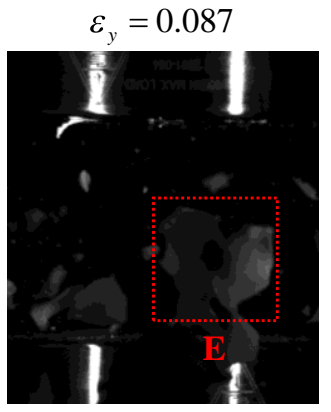

E

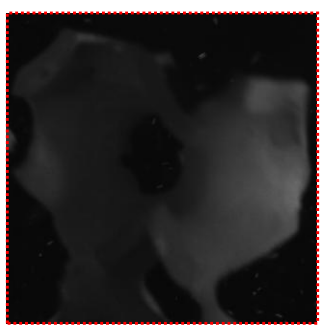

$\varepsilon_{y}=0.58$

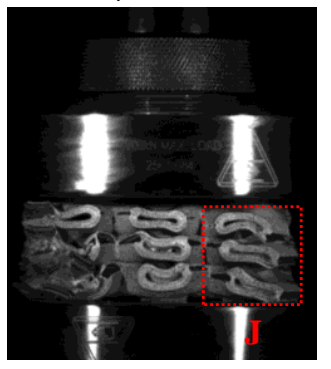

J

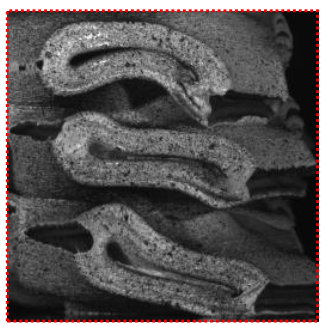

Figure 3. Mechanical deformation of the 3D printed VW and MCC structures. (a) and (b) Global deformation and corresponding local deformation of 3D printed VW structure at different strain levels, respectively. (c) and (d) Global deformation and corresponding local deformation of 3D printed MCC configurations at different strain levels, respectively. 


\subsection{Finite element simulations}

To further understand the underlying deformation mechanisms related to these structures, finite element (FE) simulations have been performed on both the VW and the MCC structures. A user-defined viscoplastic model for the VeroWhitePlus and a hyperelastic model for Agilus30 have been implemented in a commercial finite element code. Figures 4 (a) and (d) show the comparison between measured and simulated stress-strain relations for the VW and the MCC structures, respectively. The experimental results and the FE simulations are in good quantitative agreement. Very good correlation is evident between experiments and simulations for the VW structure for strains lower than $8 \%$. For the MCC structure, the two sets of stress-strain curves compare well for compressions lower than 10\%. Furthermore, the large experimental deformation exhibited by the MCC structure also coincides very well with the FE simulation results for compressions lower than $40 \%$. After this level of strain, the discrepancy between simulations and experiments begins to be significant as densification begins to occur. The main rationale behind the difference between experiments and simulations is the lack of incorporation of failure in the FE models. Nevertheless, the good agreement between experiments and simulations indicate that the FE modeling approach used here can predict the nonlinear mechanical behavior of the cellular structures within a sizeable compressive strain range.

Two-dimensional DIC measurements were also taken to identify the strain patterns of the two configurations during the mechanical testing. The DIC results of the VW and MCC configurations under different strain levels are displayed in Figure S2 (SI), and the corresponding strain and stress evolution of the contour plots from the FE simulations can be found in Figure S3 (SI). The experimental and simulated strain distributions at a global strain level of 5\% for the VW structure are displayed in Figures 4 (b) and (c). The FE simulations 
overpredict the magnitudes of the maximum tensile and compressive strain concentrations. This is because the 2D DIC system used here may not be able to accurately capture the strain distribution in the complex surfaces. Nevertheless, the local strain measurements and the FE predictions are in good qualitative agreement, with distinctive thin horizontal bands of tensile strain near the center of each cell. It is noteworthy that the catastrophic failure of the VW structure (see Figure 3 (a)) initiated due to cracks forming in the banded tensile strain region shown in Figures 4 (b) and (c).

Figures 4 (e) and 4 (f) show the comparison between the DIC measurements and FE simulation results for the MCC structure at a global strain level of 5\%. The MCC configuration does not feature the vertical strain concentrated in a small region, rather it is distributed evenly around the contact areas between unit cells along both the horizontal and vertical directions. The MCC configuration however shows a significantly more uniform stress distribution. This uniform stress distribution phenomenon indicates that the synergistic interplay between the two compositions acts an important way in suppressing rapid crack propagation and leading to a progressive failure behavior. In particular, the soft phase functions as a spring to absorb part of the energy during compression, resulting in more ductile behavior. Consequently, the MCC configuration exhibits the superior energy absorption capability under large deformations. 
(a)

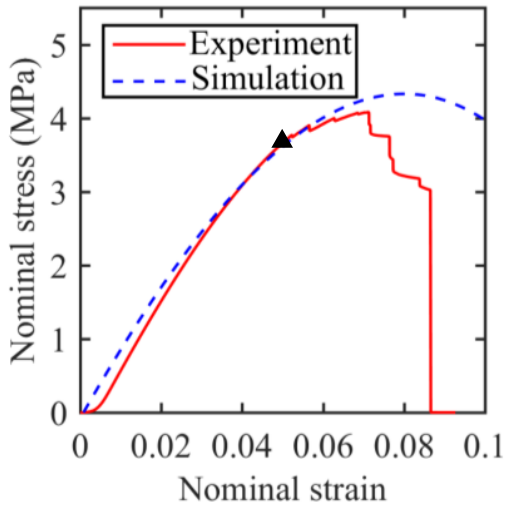

(d)

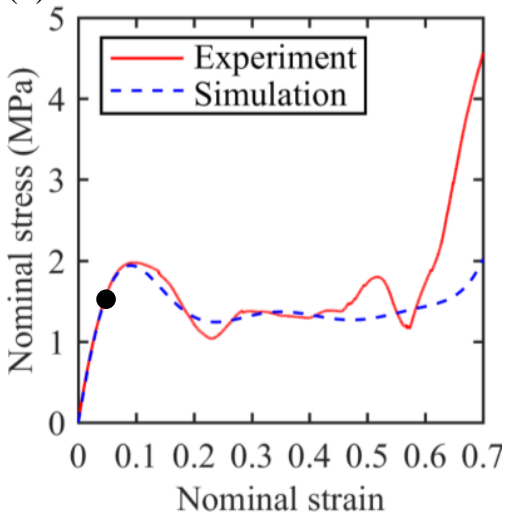

(b) Experiment

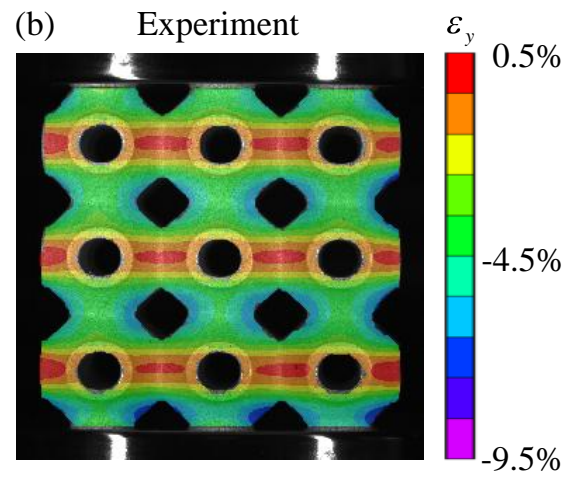

(e)

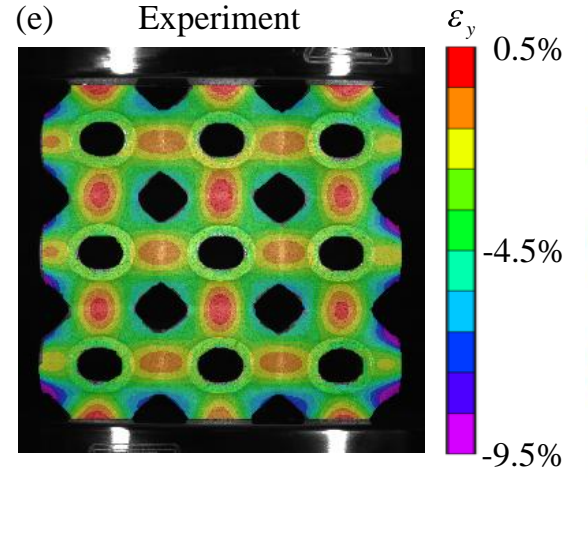

(c) Simulation

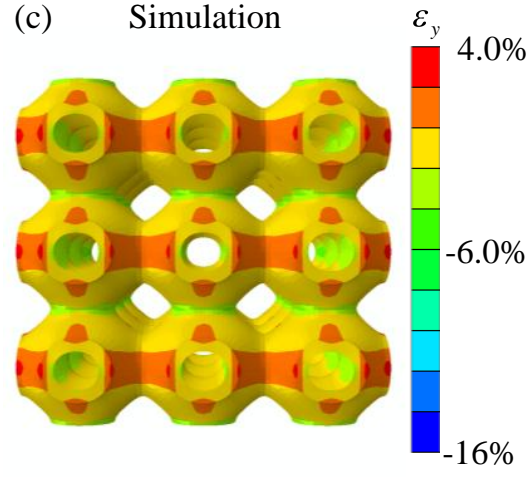

(f)

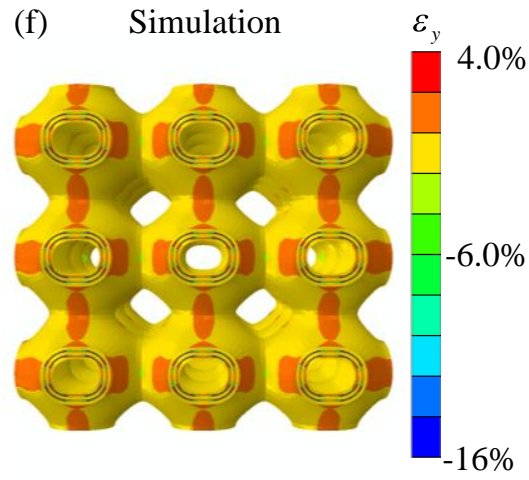

Figure 4. Digital image correlation (DIC) characterization and finite element (FE) modeling. (a) Comparison of the stress-strain relations from the mechanical testing and the FE simulations for the VW structure. (b)-(c) Comparison between measured and simulated strain distributions for the VW structure. (d) Comparison of the stress-strain curves from experiments and FE simulations for the MCC configuration. (e)-(f) Comparison between measured and simulated strain distributions for MCC configuration. Here a common 5\% of compressive strain is chosen for both the VW and MCC structures.

\subsection{Shape recovery under loading-unloading condition}

Having explored the mechanical behavior and intrinsic deformation mechanisms of the MCC topologies, we proceed to study their structural integrity and shape recoverability. Cyclic loading tests on the MCC structures were performed for five cycles up to strains of $20 \%$ and $40 \%$, 
respectively. Figure 5 (a) shows the stress-strain relations of the MCC structure when compressed up to $20 \%$. For the first cycle, the structure can fully recover its initial form (Figure 5 (i)). Even when failure initiates and propagates, the structure can recover to the initial shape gradually when unloaded. This behavior is due to the synergistic interplay between the multilayered architecture and the two compositions involved. The soft phase in particular acts like a spring between hard layers that absorbs the elastic energy during compression and releases the stored elastic energy when the load is removed.

To accelerate the cyclic tests, each compressed sample after the first cycle was heated up to 75 ${ }^{\circ} \mathrm{C}$ for 10 minutes. The samples can recover their initial form due to the shape memory effect of the VeroWhitePlus. It should be pointed out that the materials we used here are thermoplastics [71], implying that they can be heated and freely remolded without changing their physical properties. The heated samples cooled down afterward to room temperature in 30 minutes. Cyclic tests and shape memory assisted recovery were then performed on the samples at maximum compressible strains of $20 \%$ and $40 \%$, respectively. Figure 5 (b) shows that the effective stiffness of the MCC structure decreases with the number of cycles. This is because the fracture and failure zones increase and accumulate with the increase of the cycle number, similarly to the Mullins effect in porous solids (Figure S4) [72]. For the $2^{\text {nd }}, 3^{\text {rd }}, 4^{\text {th }}$, and $5^{\text {th }}$ cycles, the stiffness decreased by $23.8 \%, 25.9 \%, 29.6 \%$, and $51.1 \%$, respectively. Figure 5 (c) shows the maximum stresses at different cycles. The maximum stress, in this case, decreases from the $1^{\text {st }}$ to the $4^{\text {th }}$ cycle but then increases between the $4^{\text {th }}$ and $5^{\text {th }}$. This is because the failure zone enlarges with the increase of the number of cycles, and this lowers the first peak stress and postpones the maximum stress point to the subsequent densification range. The energy dissipation ratio is calculated as the energy dissipation divided by energy absorption, where 
energy dissipation is defined as the area enclosed by the loading and unloading stress-strain curve. Energy dissipation ratio is plotted in Figure 5 (d). The energy dissipation ratio slightly changes during the different cycles, but all are between 0.8 and 0.9 , indicating that most of the energy input during the compressive loading phase is efficiently dissipated. It should be pointed out that, though the energy dissipation decreased for the $2^{\text {nd }}$ cycle, the energy absorption decreased as well. This is why the energy dissipation ratio counterintuitively increased compared with the $1^{\text {st }}$ cycle.

Figure 5 (e) shows the stress-strain curve of the MCC configuration when compressed up to 40\%. Once again, the MCC structure can recover its initial shape due to the same intrinsic deformation mechanisms (Figure $5(\mathbf{j})$ ). The stiffness decreases quite remarkably from the $1^{\text {st }}$ to $5^{\text {th }}$ cycle by $42.7 \%, 67.9 \%, 97.1 \%$, and $98.5 \%$, respectively (Figure 5 (f)). The reason for this rapid decrease is the generation of cracks and the enlarging of the failure zone accumulated in the structure with the increase of the number of cycles (Figure S5). The maximum stress shown in Figure 5 (g) decreases by $\sim 35 \%$ and then remains constant for the other cycles. Similarly, the energy dissipation ratio slightly decreases between the $1^{\text {st }}$ and the $5^{\text {th }}$ cycles with a stable value around 0.9, which indicates that the MCC structure is an excellent energy absorber (Figure 5 (h)). These cyclic loading tests indicate that the proposed MCC configuration possesses excellent structural integrity and remarkable shape recoverability under large deformations due to the mutual bonding between hard and soft phases that can prevent catastrophic failure under compression. The compressive energy is partially dissipated by the viscoplastic deformation and fracture of the hard, brittle phase, while the soft phase, though only has a small fraction of the volume, can store considerable elastic energy. This stored strain elastic energy makes the soft 
phase act similarly to an elastic spring, which is essential for the shape recovery of the MCC structure during the unloading stage.
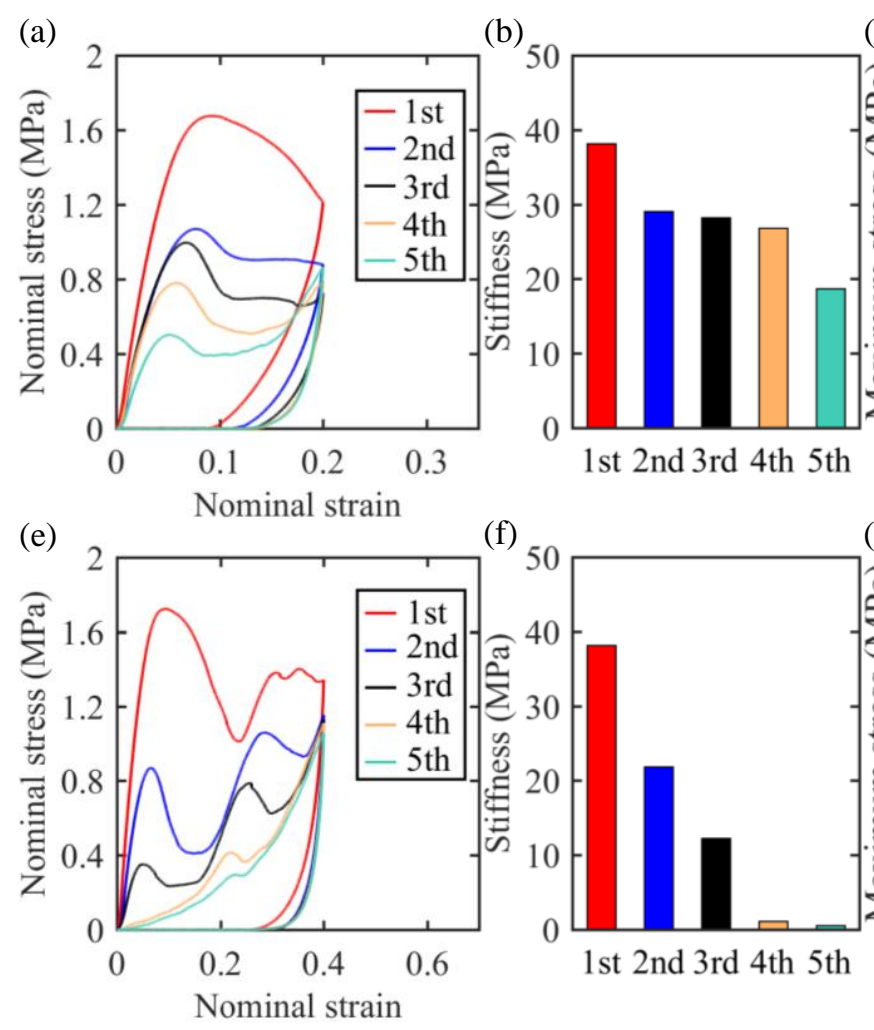

(f)
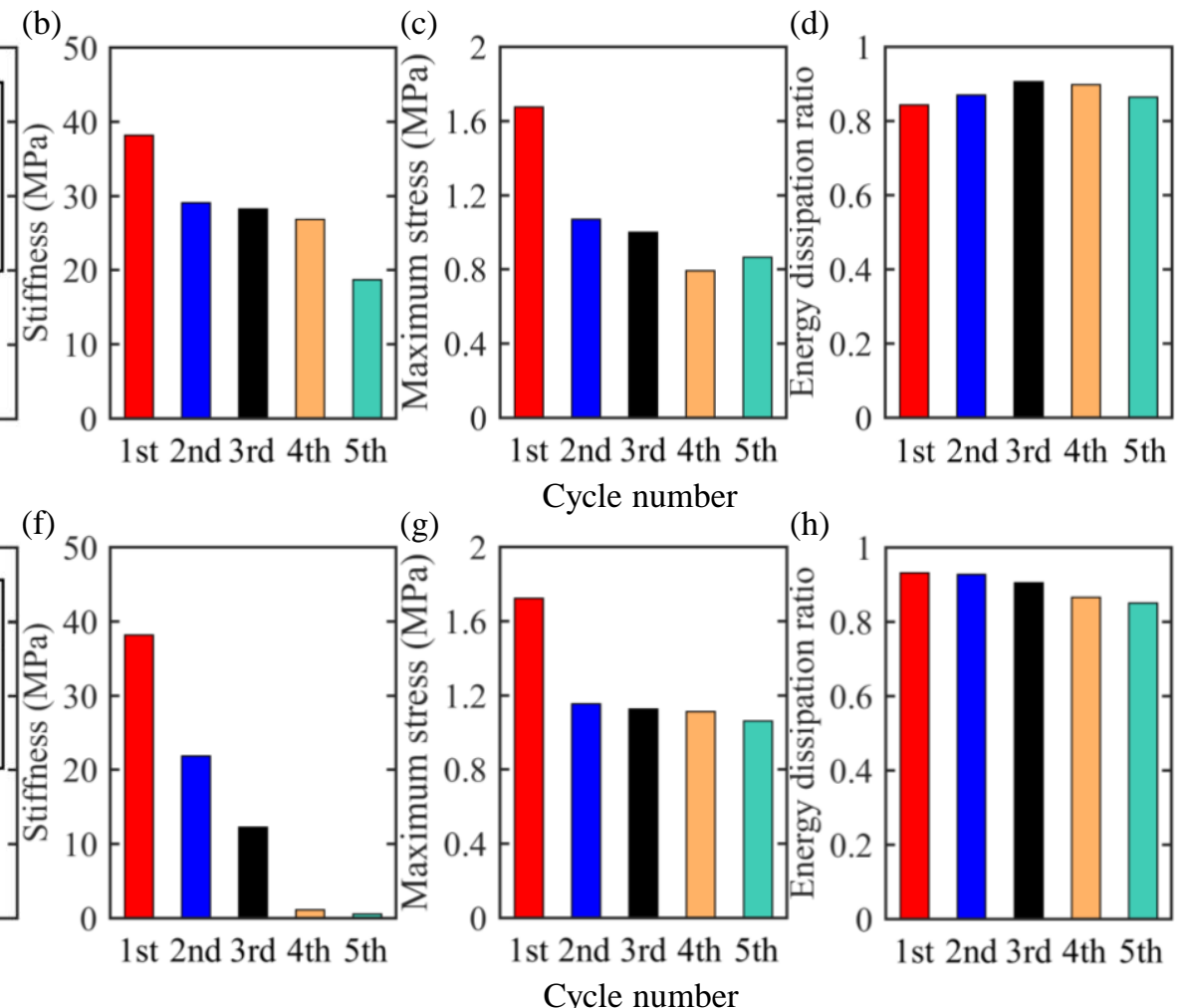

(h)

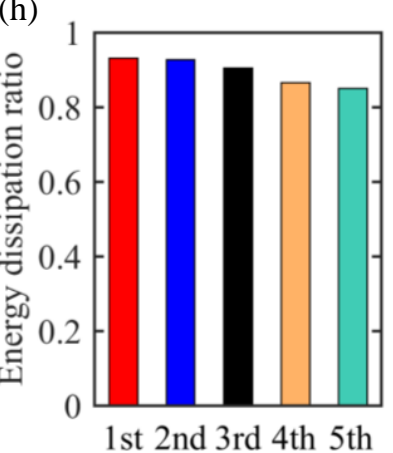

(i)

$\varepsilon_{v}=0.12$

$\varepsilon_{y}=0.2$
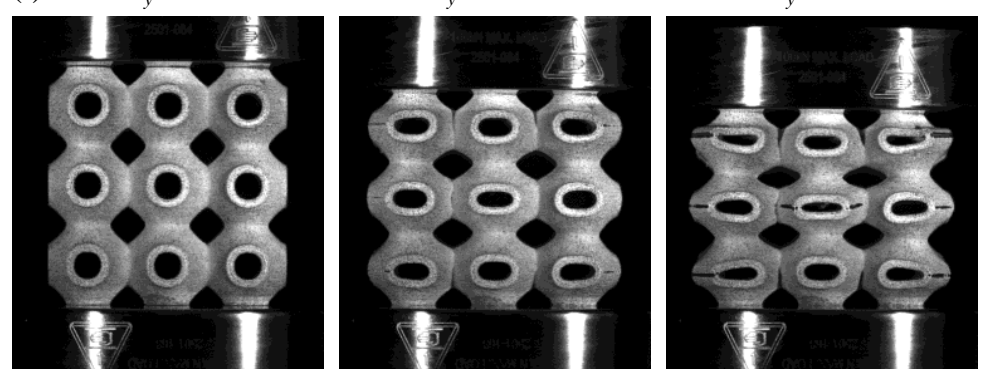

$\varepsilon_{y}=0.12$

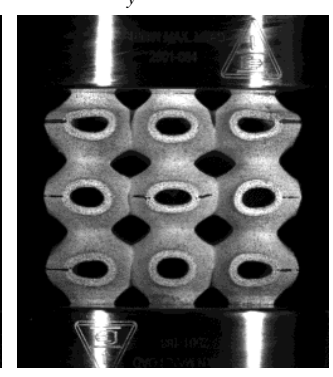

$\varepsilon_{y}=0$

(j)

$\varepsilon_{y}=0.27$

$\varepsilon_{y}=0.4$
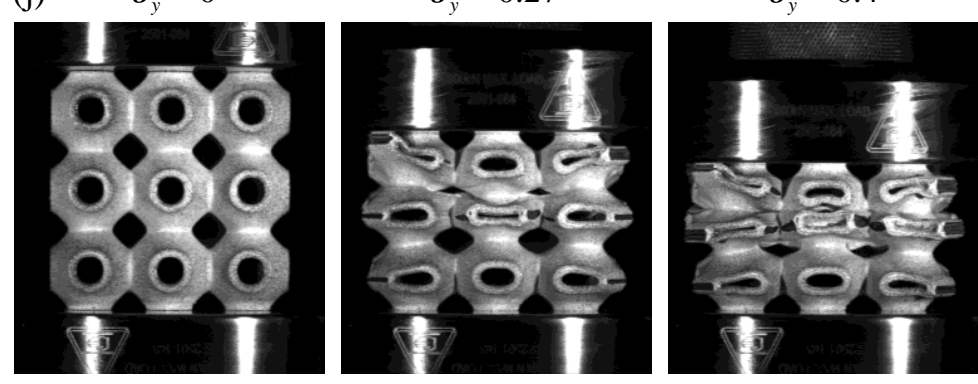

$\varepsilon_{y}=0.27$
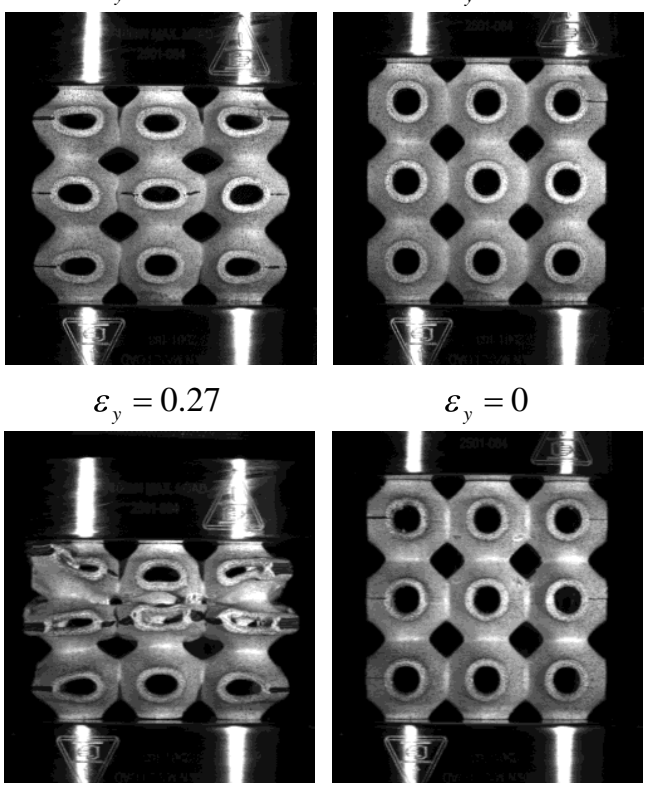

$\varepsilon_{y}=0$

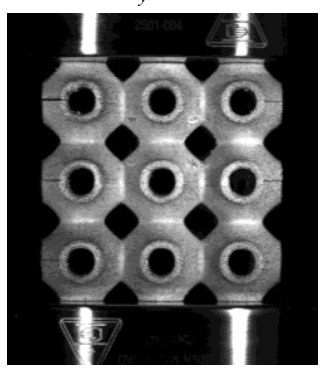


Figure 5. Mechanical properties of the MCC configurations under cyclic loading tests. (a)-(d) Stress-strain curves, stiffness, maximum stresses, and energy diss ipation ratios at different cycles when the structure is compressed up to $20 \%$. (e)-(h) Stress-strain curves, stiffness, maximum stresses, and energy dissipation ratios at different cycles when the MCC configuration is compressed up to $40 \%$. (i)-(j) Deformation of the MCC structures at different strain levels when the MCC structure is compressed up to $20 \%$ and $40 \%$ after the first cycle, respectively.

\subsection{Role of soft phase}

It is clear that the soft phase in the MCC structures plays a pivotal role in the energy absorption and shape recoverability of these materials. To further quantify the role of the soft phase on the mechanical performance, study has been performed on the effect of soft phase volume fraction (Vf). Here four additional samples with soft phase Vf of $10 \%, 30 \%$, 40\%, and $50 \%$ have been designed, printed, and compressed up to $70 \%$. One should notice that all the structures investigated here have have approximately the same mass, as the densities of VeroWhitePlus and Agilus30 are $1170 \mathrm{~kg} / \mathrm{m}^{3}$ and $1150 \mathrm{~kg} / \mathrm{m}^{3}$, respectively. The stress-strain curve for each Vf is shown in Figure 6 (a). Beyond the $10 \%$ of compressive strain, the stress drops rapidly for the MCC structure with $\mathrm{Vf}=10 \%$. In this case, the soft phase fraction is so small that it cannot absorb enough elastic energy, and this gives rise to a brittle-like deformation behavior. The onset of failure begins to occur at the corners of the structure at $15 \%$ of compressive strain (Figure 6 (e)) and the top layer fails in a brittle mode at $25 \%$. The failure zone then expands to the bottom layer and the whole structure when the strain reaches deformations of $40 \%$ and $50 \%$. No obvious densification is present due to the subsequent catastrophic failure of the structure. With the increase of the volume fraction, the MCC structure exhibits a stable and progressive failure mode. For example, when the volume fraction of the soft 
phase increases to 0.5 the stress-strain curve shows a long and constant stress plateau between $5 \%$ and $60 \%$ of compression, followed by noticeable densification. This stress evolution indicates a progressive failure mode, as highlighted by the deformation patterns shown in Figure 6 (f). No visible failure is present within the structure before $25 \%$ of compression, and the structure releases strain energy through buckling instead of fracture due to the significant portion of the soft phase. The structure then fractures in a small portion of the volume while maintaining overall integrity at a strain of $40 \%$ and $50 \%$.

The stiffness, strength and energy absorption of the MCC structures with various soft phase volume fractions are summarized in Figures 6 (b)-(d). As expected, both stiffness and strength decrease with the increase of the soft phase volume fraction. The energy absorption increases only slightly when the volume fraction changes from 0.1 to 0.2 , and this is attributed to the different failure modes present in the two different MCC structures. The energy absorption decreases significantly when the volume fraction increases up to 0.5 to a final value of 0.26 $\mathrm{MJ} / \mathrm{m}^{3}$, which is comparable to the energy absorption exhibited by the VW structure $(0.27$ $\mathrm{MJ} / \mathrm{m}^{3}$ ). This indicates that when the volume fraction of the soft phase exceeds 0.5 , it is not possible to obtain further energy absorption increases because of the decreases in stiffness and strength. 

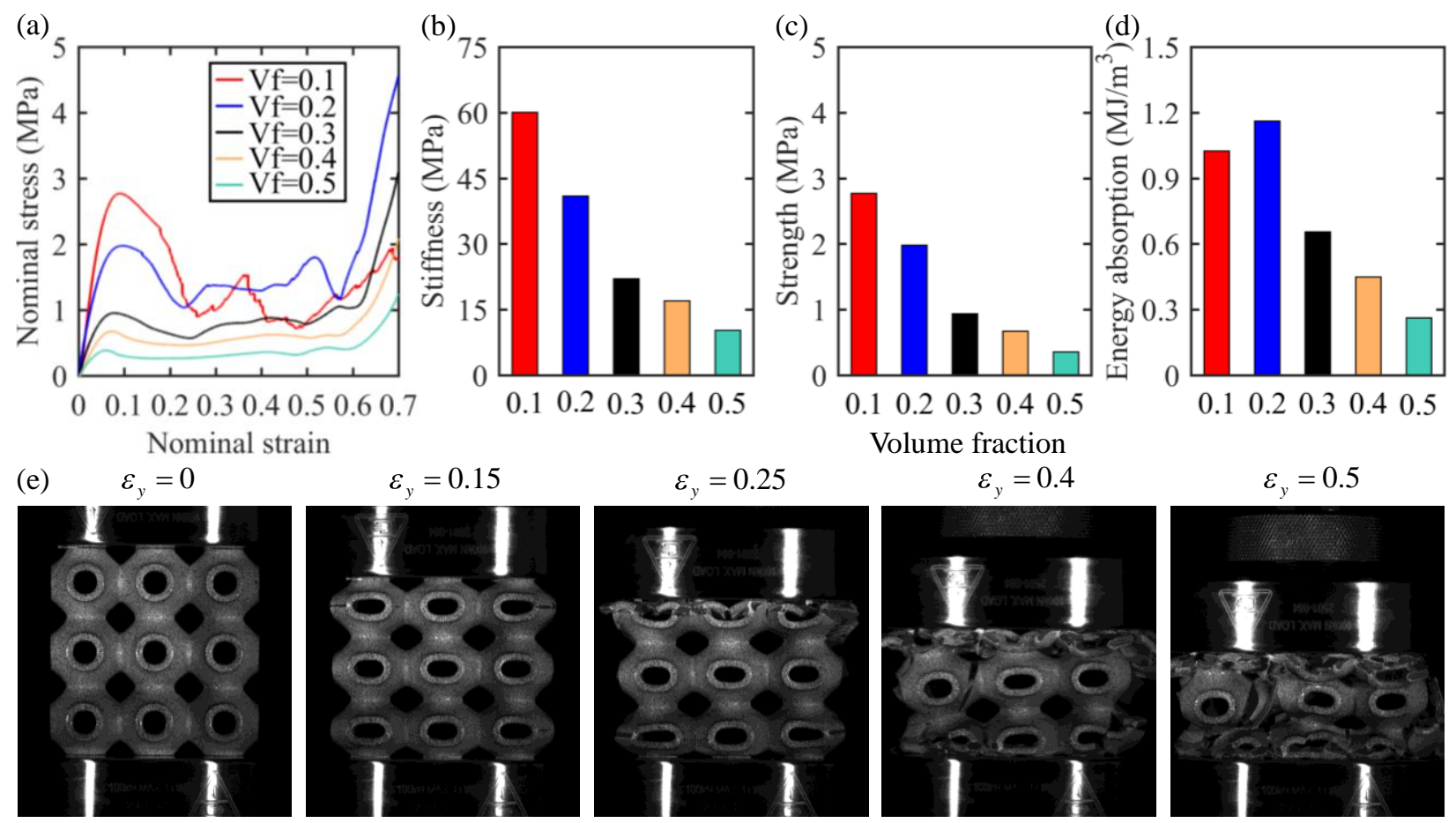

(f) $\varepsilon_{y}=0.15$ $\varepsilon_{y}=0.25$
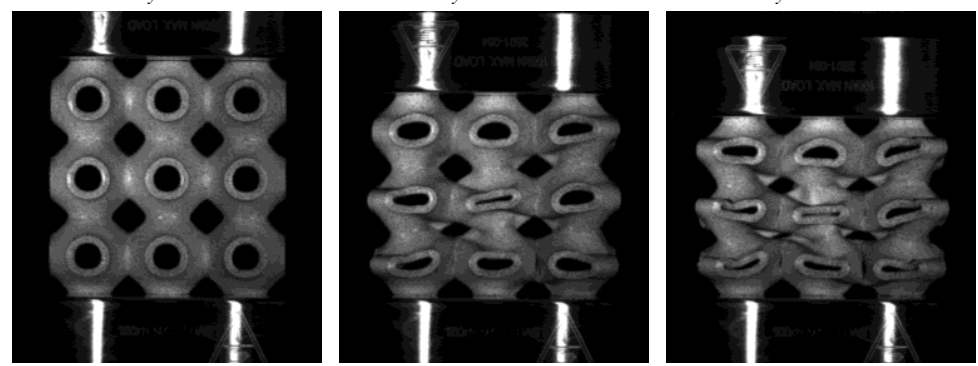

$\varepsilon_{y}=0.4$
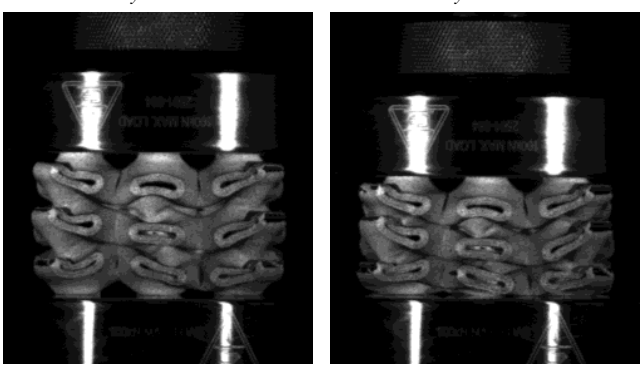

Figure 6. Effect of the volume fraction of the soft phase on the mechanical response of the MCC structures. (a)-(d) Stress-strain curves, stiffness, strength, and energy absorption at different volume fractions, respectively. (e)-(f) Deformation patterns of the MCC structures with a soft phase volume fraction of $10 \%$ and $50 \%$, respectively.

\section{Conclusions}

In summary, this paper presents the investigation of the mechanical response of a new type of 3D printed, bioinspired, cellular composite through a combined experimental and numerical effort. Compressive mechanical tests carried out on the 3D printed VW and MCC structures suggest that the multilayered architecture used in this work can turn an original brittle, 
catastrophic failure mode into a progressive one, resulting in enhanced energy absorption capabilities under large deformations. Finite element simulations along with 2D DIC measurements reveal that the mutual constraint and interaction between the two material phases, along with the microstructure of the MCC structure, are responsible for this enhanced mechanical performance. In addition, the MCC structures exhibit excellent and remarkable shape recoverability under a compressive strain up to $40 \%$, which is ascribed to the synergistic interplay between the two phases in the multilayered architecture. The enhanced energy absorption of the proposed cellular composites makes them ideal candidates for structures whose designs are controlled by crash or impact load cases. The remarkable shape recoverability is a feature that could be used to design novel morphing structures and soft robotics devices. Nevertheless, Increasing energy absorption is often accompanied by the reduction of stiffness or strength. This issue can be remediated by using advanced modeling frameworks such as topology optimization or machine learning algorithms to optimize the bala nce between energy absorption and stiffness or strength. Although the $3 \mathrm{D}$ printed system evaluated in this work has features at the millimeter scale due to the resolution of the additive manufacturing equipment used, the design principles and the working mechanisms are transferable to other architected cellular materials across multiple length scales. The findings discovered here can be used as useful guidelines to novel develop lightweight composite architectures for various energy absorption

engineering applications in automotive, aerospace vehicles, personal protective structures in sports, and lightweight package materials for the semiconductor and energy industry.

\section{Supporting information}

Supporting information is provided at the end of this manuscript. 


\section{Author contributions}

The manuscript was written through the contributions of all authors. All authors have approved the final version of the manuscript.

\section{Acknowledgment}

YC gratefully acknowledges the start-up fund from the Department of Mechanical Engineering at the University of Louisville

\section{REFERENCES}

[1] G. Cole, A. Sherman, Light weight materials for automotive applications, Mater. Charact. 35 (1) (1995) 3-9.

[2] W.J. Joost, Reducing vehicle weight and improving US energy efficiency using integrated computational materials engineering, JOM. 64 (9) (2012) 1032-1038.

[3] S. Amjad, S. Neelakrishnan, R. Rudramoorthy, Review of design considerations and technological challenges for successful development and deployment of plug-in hybrid electric vehicles, Renew. Sust. Energ. Rev. 14 (3) (2010) 1104-1110.

[4] S. Kobayashi, S. Plotkin, S.K. Ribeiro, Energy efficiency technologies for road vehicles, Energ. Effic. 2 (2) (2009) 125-137.

[5] G. Lu, Energy absorption requirement for crashworthy vehicles, P. I. Mech. Eng. F-J. Rai. 216 (1) (2002) 31-39.

[6] J. Banhart, Aluminium foams for lighter vehicles, Int. J. Vehicle Des. 37 (2-3) (2005) 114125.

[7] P. Griškevičius, A. Žiliukas, The crash energy absorption of the vehicles front structures, Transport. 18 (2) (2003) 97-101.

[8] P. Xu, C. Yang, Y. Peng, S. Yao, D. Zhang, B. Li, Crash performance and multi-objective optimization of a gradual energy-absorbing structure for subway vehicles, Int. J. Mech. Sci. 107 (2016) 1-12.

[9] S.S. Cheon, K.S. Jeong, Composite side-door impact beams for passenger cars, Compos. Struct. 38 (1-4) (1997) 229-239.

[10] R. Hosseinzadeh, M.M. Shokrieh, L.B. Lessard, Parametric study of automotive composite bumper beams subjected to low-velocity impacts, Compos. Struct. 68 (4) (2005) 419-427.

[11] U.G. Wegst, H. Bai, E. Saiz, A.P. Tomsia, R.O. Ritchie, Bioinspired structural materials, Nat. Mater. 14 (1) (2015) 23.

[12] C. Ortiz, M.C. Boyce, Bioinspired structural materials, Science. 319 (5866) (2008) 1053 1054.

[13] H. Gao, B. Ji, I.L. Jäger, E. Arzt, P. Fratzl, Materials become insensitive to flaws at nanoscale: lessons from nature, PNAS. 100 (10) (2003) 5597-5600. 
[14] P. Tran, T.D. Ngo, A. Ghazlan, D. Hui, Bimaterial 3D printing and numerical analysis of bio-inspired composite structures under in-plane and transverse loadings, Compos. Part B-Eng. 108 (2017) 210-223.

[15] L.S. Dimas, G.H. Bratzel, I. Eylon, M.J. Buehler, Tough composites inspired by mineralized natural materials: computation, 3D printing, and testing, Adv. Funct. Mater. 23 (36) (2013) 4629-4638.

[16] L.S. Dimas, M.J. Buehler, Modeling and additive manufacturing of bio-inspired composites with tunable fracture mechanical properties, Soft Matter. 10 (25) (2014) 4436-4442.

[17] M. Grossman, D. Pivovarov, F. Bouville, C. Dransfeld, K. Masania, A.R. Studart, Hierarchical Toughening of Nacre-Like Composites, Adv. Funct. Mater. 29 (9) (2019) 1806800. [18] M. Grossman, F. Bouville, F. Erni, K. Masania, R. Libanori, A.R. Studart, Mineral nanointerconnectivity stiffens and toughens nacre-like composite materials, Adv. Mater. 29 (8) (2017) 1605039.

[19] A. Woesz, J.C. Weaver, M. Kazanci, Y. Dauphin, J. Aizenberg, D.E. Morse, P. Fratzl, Micromechanical properties of biological silica in skeletons of deep-sea sponges, J. Mater. Res. 21 (8) (2006) 2068-2078.

[20] G. Mayer, R. Trejo, E. Lara-Curzio, M. Rodriguez, K. Tran, H. Song, W. Ma, Lessons for new classes of inorganic/organic composites from the spicules and skeleton of the sea sponge Euplectella aspergillum, OPL. 844 (2004).

[21] M.E. Birkbak, M. Guizar-Sicairos, M. Holler, H. Birkedal, Internal structure of sponge glass fiber revealed by ptychographic nanotomography, J. Struct. Biol. 194 (1) (2016) 124-128.

[22] J.C. Weaver, J. Aizenberg, G.E. Fantner, D. Kisailus, A. Woesz, P. Allen, K. Fields, M.J. Porter, F.W. Zok, P.K. Hansma, Hierarchical assembly of the siliceous skeletal lattice of the hexactinellid sponge Euplectella aspergillum, J. Struct. Biol. 158 (1) (2007) 93-106.

[23] H. Gao, Application of fracture mechanics concepts to hierarchical biomechanics of bone and bone-like materials, Int. J. Fract. 138 (1-4) (2006) 101.

[24] A.R. Studart, Towards high-performance bioinspired composites, Adv. Mater. 24 (37) (2012) 5024-5044.

[25] P. Chen, A. Lin, Y. Lin, Y. Seki, A. Stokes, J. Peyras, E. Olevsky, M. Meyers, J. McKittrick, Structure and mechanical properties of selected biological materials, J. Mech. Behav. Biomed. 1 (3) (2008) 208-226.

[26] S. Bechtle, S.F. Ang, G.A. Schneider, On the mechanical properties of hierarchically structured biological materials, Biomaterials. 31 (25) (2010) 6378-6385.

[27] L.J. Gibson, M.F. Ashby, G. Schajer, C. Robertson, The mechanics of two-dimensional cellular materials, P. Roy. Soc A-Math. Phy. 382 (1782) (1982) 25-42.

[28] L.J. Gibson, M.F. Ashby, Cellular solids: structure and properties, Cambridge university, 1999.

[29] P. Baranowski, P. Płatek, A. Antolak-Dudka, M. Sarzyński, M. Kucewicz, T. Durejko, J. Małachowski, J. Janiszewski, T. Czujko, Deformation of honeycomb cellular structures manufactured with Laser Engineered Net Shaping (LENS) technology under quasi-static loading: Experimental testing and simulation, Addit. Manuf. 25 (2019) 307-316.

[30] A.G. Evans, J.W. Hutchinson, N.A. Fleck, M. Ashby, H. Wadley, The topological design of multifunctional cellular metals, Prog. Mater. Sci. 46 (3-4) (2001) 309-327.

[31] Y. Du, H. Li, Z. Luo, Q. Tian, Topological design optimization of lattice structures to maximize shear stiffness, Adv. Eng. Softw. 112 (2017) 211-221. 
[32] M. Kucewicz, P. Baranowski, J. Małachowski, A. Popławski, P. Płatek, Modelling, and characterization of 3D printed cellular structures, Mater. Des. 142 (2018) 177-189.

[33] S.R. Bates, I.R. Farrow, R.S. Trask, 3D printed polyurethane honeycombs for repeated tailored energy absorption, Mater. Des. 112 (2016) 172-183.

[34] D. Li, W. Liao, N. Dai, Y.M. Xie, Comparison of mechanical properties and energy absorption of sheet-based and strut-based gyroid cellular structures with graded densities, Materials. 12 (13) (2019) 2183.

[35] M.S. Rad, H. Hatami, R. Alipouri, A.F. Nejad, F. Omidinasab, Determination of energy absorption in different cellular auxetic structures, Mech ind. 20 (3) (2019) 302.

[36] B.G. Compton, J.A. Lewis, 3D-printing of lightweight cellular composites, Adv. Mater. 26 (34) (2014) 5930-5935.

[37] S. Malek, J.R. Raney, J.A. Lewis, L.J. Gibson, Lightweight 3D cellular composites inspired by balsa, Bioinspir. Biomim. 12 (2) (2017) 026014.

[38] Y. Chen, T. Li, Z. Jia, F. Scarpa, C. Yao, L. Wang, 3D printed hierarchical honeycombs with shape integrity under large compressive deformations, Mater. Design. 137 (2018) 226-234. [39] L.J. Gibson, K.E. Easterling, M.F. Ashby, The structure and mechanics of cork, P. Roy. Soc A-Math. Phy. 377 (1769) (1981) 99-117.

[40] R. Hooke, Micrographia (1665), AppLife, 2014.

[41] L. Le Barbenchon, J. Girardot, J.-B. Kopp, P. Viot, Multi-scale foam: 3D structure/compressive behaviour relationship of agglomerated cork, Materialia. 5 (2019) 100219. [42] F.C. Potes, J.M. Silva, P.V. Gamboa, Development and characterization of a natural lightweight composite solution for aircraft structural applications, Compos. Struct. 136 (2016) 430-440.

[43] S.P. Silva, M.A. Sabino, E.M. Fernandes, V.M. Correlo, L.F. Boesel, R.L. Reis, Cork: properties, capabilities and applications, Int. Mater. Rev. 50 (6) (2005) 345-365.

[44] X. Song, J. Zhao, Ultrastructural study of plasmodesmata in cork cells from Quercus variabilis Blume (Fagaceae), Ind. Crop Prod. 97 (2017) 275-280.

[45] H. Pereira, The rationale behind cork properties: a review of structure and chemistry, Bioresources. 10 (3) (2015) 6207-6229.

[46] S. Silva, M. Sabino, E. Fernandes, V. Correlo, L. Boesel, R. Reis, Cork: properties, capabilities and applications, Int. Mater. Rev. 50 (6) (2005) 345-365.

[47] H. Pereira, Chemical composition and variability of cork from Quercus suber L, Wood Sci. Technol. 22 (3) (1988) 211-218.

[48] O. Al-Ketan, R. Rowshan, R.K.A. Al-Rub, Topology-mechanical property relationship of 3D printed strut, skeletal, and sheet based periodic metallic cellular materials, Additive Manufacturing. 19 (2018) 167-183.

[49] C. Bonatti, D. Mohr, Smooth-shell metamaterials of cubic symmetry: anisotropic elasticity, yield strength and specific energy absorption, Acta Materialia. 164 (2019) 301-321.

[50] M. Maldovan, C.K. Ullal, J.H. Jang, E.L. Thomas, Sub-Micrometer Scale Periodic Porous Cellular Structures: Microframes Prepared by Holographic Interference Lithography, Adv. Mater. 19 (22) (2007) 3809-3813.

[51] S. Kramer, J.L. Jordan, H. Jin, J. Carroll, A.M. Beese, Mechanics of Additive and Advanced Manufacturing, Volume 8: Proceedings of the 2018 Annual Conference on Experimental and Applied Mechanics, Springer, 2018.

[52] ASTM D638-14, Standard test method for tensile properties of plastics, 2014. 
[53] ASTM D412, Standard test methods for VulCanized rubber and thermoplastic rubbers and thermoplastic elastomers, 1998.

[54] C. Dascalu, G. Bilbie, E. Agiasofitou, Damage and size effects in elastic solids: a homogenization approach, Int. J. Solids Struct. 45 (2) (2008) 409-430.

[55] J.R. Jain, S. Ghosh, Damage evolution in composites with a homogenization-based continuum damage mechanics model, Int. J. Damage Mech.18 (6) (2009) 533-568.

[56] H. Talebi, M. Silani, S.P. Bordas, P. Kerfriden, T. Rabczuk, A computational library for multiscale modeling of material failure, Comput. Mech. 53 (5) (2014) 1047-1071.

[57] H. Ghasemi, H.S. Park, T. Rabczuk, A multi-material level set-based topology optimization of flexoelectric composites, Comput. Method Appl. M. 332 (2018) 47-62.

[58] E. Lund, Buckling topology optimization of laminated multi-material composite shell structures, Compos. Struct. 91 (2) (2009) 158-167.

[59] E. Samaniego, C. Anitescu, S. Goswami, V.M. Nguyen-Thanh, H. Guo, K. Hamdia, X. Zhuang, T. Rabczuk, An energy approach to the solution of partial differential equations in computational mechanics via machine learning: Concepts, implementation and applications, Comput. Method Appl. M. 362 (2020) 112790.

[60] J.-H. Song, T. Menouillard, A. Tabarraei, Explicit dynamic finite element method for failure with smooth fracture energy dissipations, Math. Probl. Eng. 2013(5) (2013) 293861.

[61] T.J. Hughes, The finite element method: linear static and dynamic finite element analysis, Courier Corporation, 2012.

[62] L. Liu, A Triaxiality-Dependent Constitutive Model For Predicting Damage-Induced Softening in 3D-Printed Soft Suture Layer, 2018.

[63] M.C. Boyce, D.M. Parks, A.S. Argon, Large inelastic deformation of glassy polymers. Part I: rate dependent constitutive model, Mech. Mater. 7 (1) (1988) 15-33.

[64] M.C. Boyce, G. Weber, D.M. Parks, On the kinematics of finite strain plasticity, J. Mech. Phys. Solids. 37 (5) (1989) 647-665.

[65] P. Zhang, A.C. To, Transversely isotropic hyperelastic-viscoplastic model for glassy polymers with application to additive manufactured photopolymers, Int. J. Plasticity. 80 (2016) 56-74.

[66] S. Zhou, T. Rabczuk, X. Zhuang, Phase field modeling of quasi-static and dynamic crack propagation: COMSOL implementation and case studies, Adv. Eng. Softw. 122 (2018) 31-49.

[67] K.M. Hamdia, M. Silani, X. Zhuang, P. He, T. Rabczuk, Stochastic analysis of the fracture toughness of polymeric nanoparticle composites using polynomial chaos expansions, Int. J. Fract. 206 (2) (2017) 215-227.

[68] V. Abaqus, 6.14 Documentation, Dassault Systemes Simulia Corporation. 651 (2014) 6.2.

[69] S. Cui, B. Gong, Q. Ding, Y. Sun, F. Ren, X. Liu, Q. Yan, H. Yang, X. Wang, B. Song, Mechanical metamaterials foams with tunable negative poisson's ratio for enhanced energy absorption and damage resistance, Materials. 11 (10) (2018) 1869.

[70] E.M. Arruda, M.C. Boyce, A three-dimensional constitutive model for the large stretch behavior of rubber elastic materials, J. Mech. Phys. Solids. 41(2) (1993) 389-412.

[71] K.-S. Noh, H.-W. Seo, Y.-G. Lee, 3D Printing Structures that Exhibit Torsions, Proceedings of the 2nd International Conference on Progress in Additive Manufacturing. (2016).

[72] C. Ge, L. Priyadarshini, D. Cormier, L. Pan, J. Tuber, A preliminary study of cushion properties of a 3D printed thermoplastic polyurethane Kelvin foam, Packag. Technol. Sci. 31 (5) (2018) 361-368. 


\section{Supporting Information}

Bioinspired multilayered cellular composites with enhanced energy absorption and shape recovery

Huan Jiang, Louise Le Barbenchon, Brett A. Bednarcyk, Fabrizio Scarpa, and Yanyu Chen*

\section{Model details}

Table S1. The parameters of the models with volume fraction from $10 \%$ to $50 \%$.

\begin{tabular}{cccccc}
\hline Volume fraction & \multicolumn{5}{c}{ Layer thickness $/ \mu m$} \\
\cline { 2 - 6 }$\%$ & 1 st & 2nd & 3rd & 4th & 5 th \\
\hline 10 & 791 & 132 & 791 & 132 & 791 \\
20 & 703 & 264 & 703 & 264 & 703 \\
30 & 615 & 395 & 615 & 395 & 615 \\
40 & 527 & 527 & 527 & 527 & 527 \\
50 & 439 & 659 & 439 & 659 & 439 \\
\hline
\end{tabular}


(a)

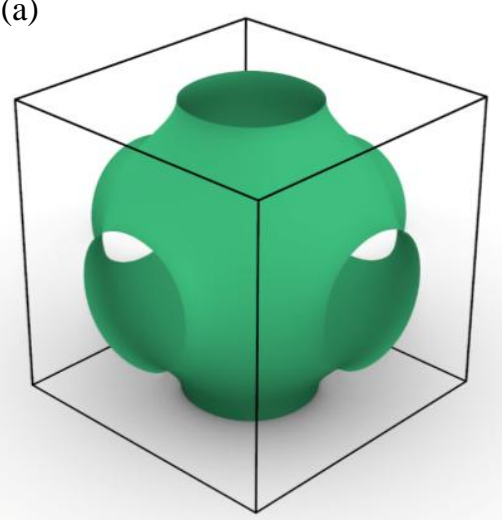

(d)

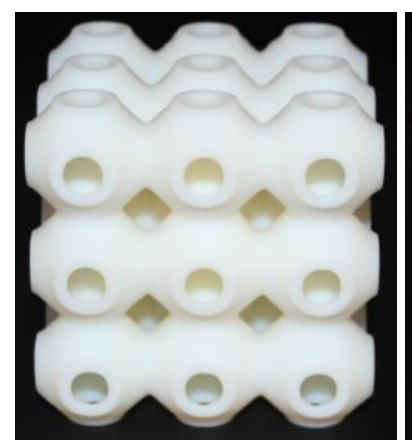

(b)

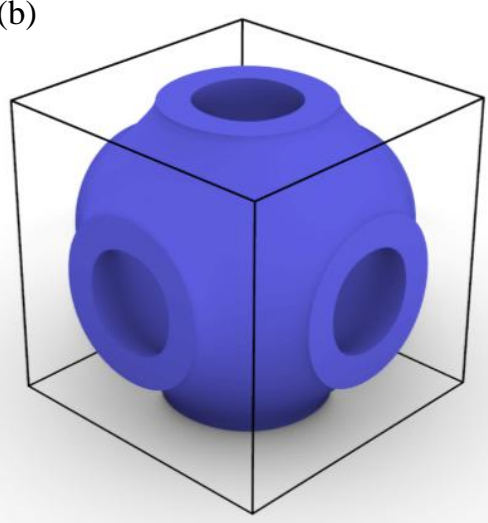

(c)

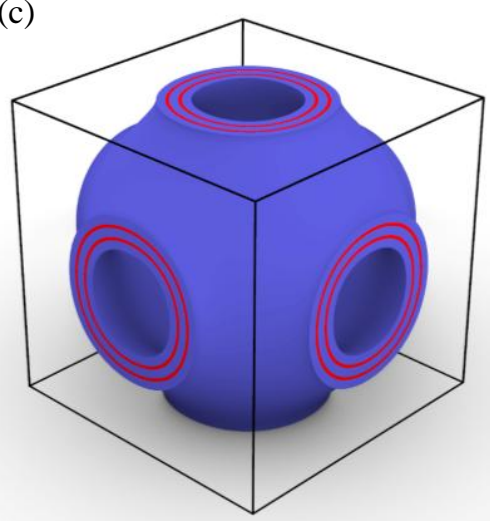

(e)
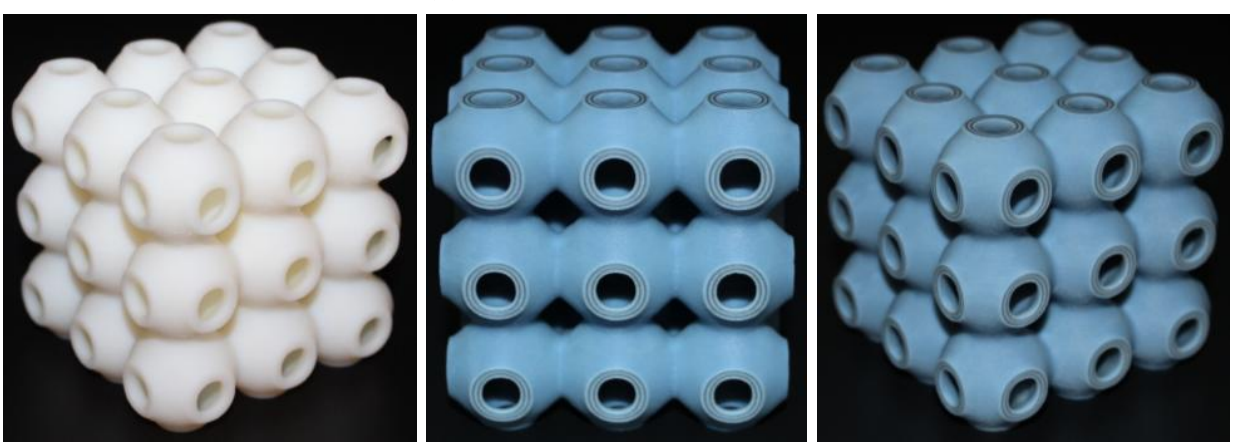

Figure S1. The design process of the proposed multilayered cellular composites. (a) Schwarz $P$ minimal surface that divides the representative volume element evenly. (b) Homogeneous VW structure with a volume fraction of $25 \%$ and (c) MCC structure with a total volume fraction of $25 \%$.The thickness of each layer from inside to outside is $703 \mu m, 264 \mu m, 703 \mu m, 264 \mu m$, and $703^{\mu m}$. (d) $3 \mathrm{D}$ printed samples for VW structure and (e) MCC structure.

\section{Additional results}

\subsection{DIC and numerical simulations for uniaxial compression tests}

Digital Image Correlation (DIC) (Correlated Solutions, USA) has been used to capture the deformation and the strain distribution of the samples during compression tests. Figure S2 (a) shows the DIC results for the VW structure at strains from 0 to $4 \%$. With the progress of the compression test, a local thin band keeps its shape and position. The strain of the VW structure, therefore, accumulates in the local area, which then leads to the local stress concentration. The 
MCC structure, however, features a more uniform distributed strain field as can be seen from Figure S2 (b). This feature is still present when the strain increases. One can even observe that the MCC architecture still exhibits a quasi-uniform strain distribution even when the structure begins to fail.

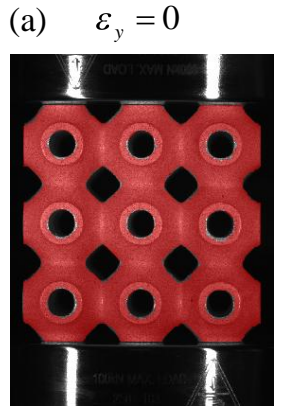

(b) $\quad \varepsilon_{y}=0$

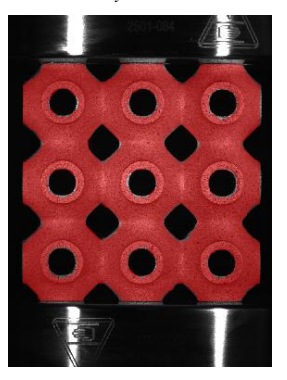

$\varepsilon_{y}=0.01$

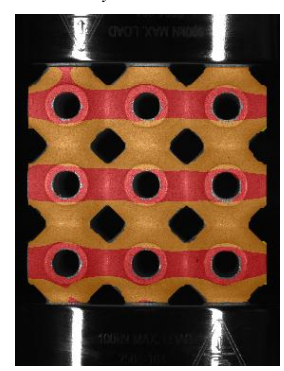

$\varepsilon_{y}=0.04$

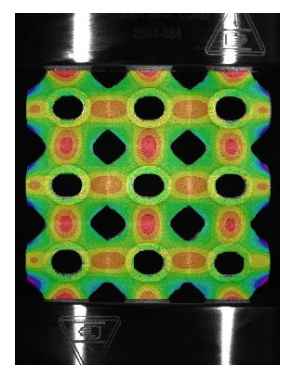

$\varepsilon_{y}=0.02$

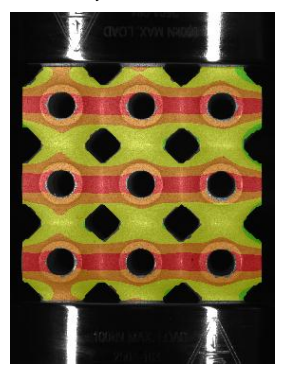

$\varepsilon_{y}=0.08$

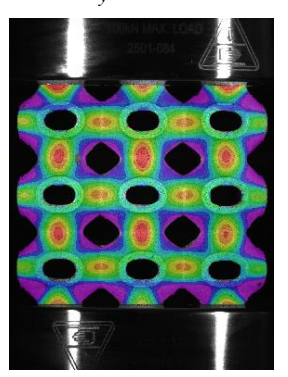

$\varepsilon_{y}=0.03$

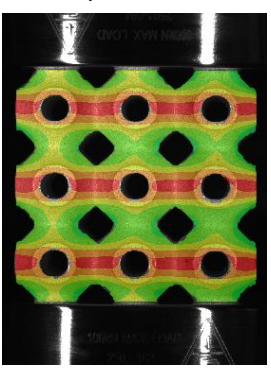

$\varepsilon_{y}=0.12$

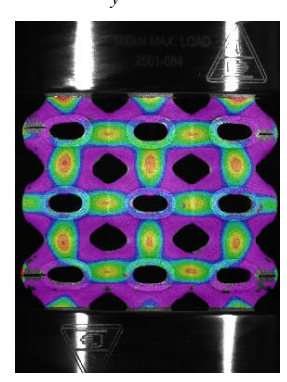

$\varepsilon_{y}=0.04$
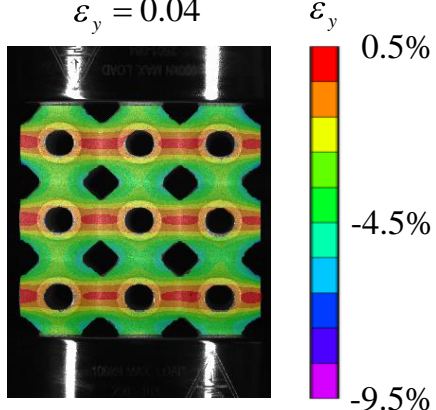

$\varepsilon_{y}=0.16$

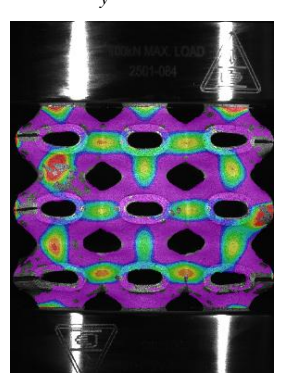

$\varepsilon_{y}$

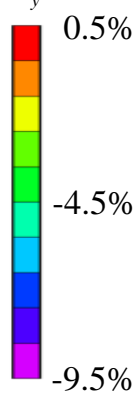

Figure S2. Measured strain evolution of the 3D printed samples. (a) VW and (b) MCC structure.

The vertical strain distribution of the $\mathrm{VW}$ and $\mathrm{MCC}$ architectures from the FE simulations is shown in Figures S3 (a) and (b), respectively. The simulated results are qualitatively consistent with those from the DIC at different strain levels. We also show the von Mises stress of the VW and MCC structures at different strains in Figures S3 (c) and (d). The von Mises stresses are concentrated at the local horizontal contact areas between unit cells for the VW configuration. The stress, however, is much more uniform around the sphere of the unit cell of the MCC structure. This further indicates that the MCC architecture has a highly efficient load transfer. 
(a) $\varepsilon_{y}=0$

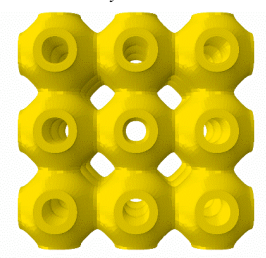

(b) $\varepsilon_{y}=0$

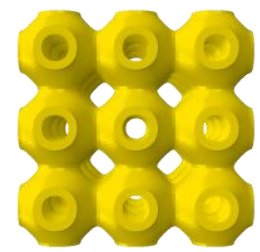

(c) $\varepsilon_{y}=0$

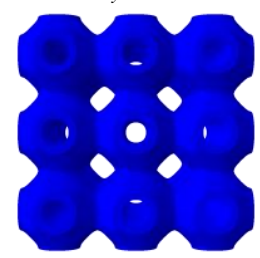

(d) $\varepsilon_{y}=0$

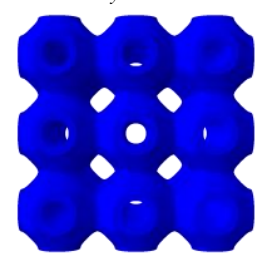

$\varepsilon_{y}=0.01$

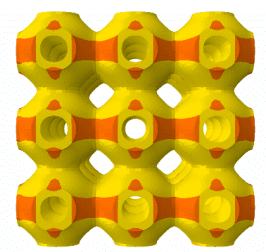

$\varepsilon_{y}=0.04$

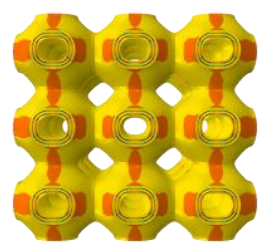

$\varepsilon_{y}=0.01$

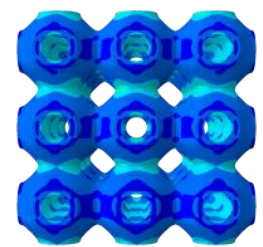

$\varepsilon_{y}=0.04$

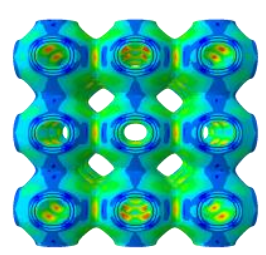

$\varepsilon_{y}=0.02$

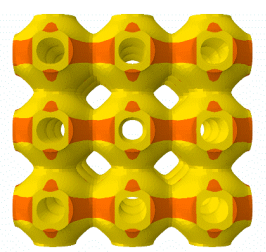

$\varepsilon_{y}=0.08$

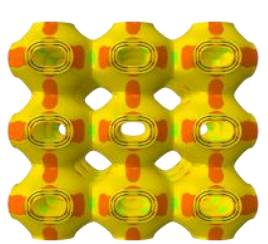

$\varepsilon_{y}=0.02$

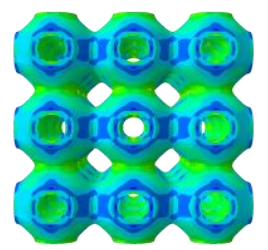

$\varepsilon_{y}=0.08$

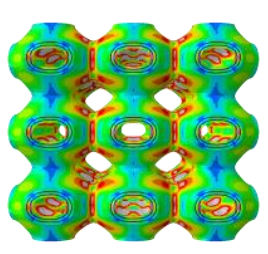

$\varepsilon_{y}=0.03$

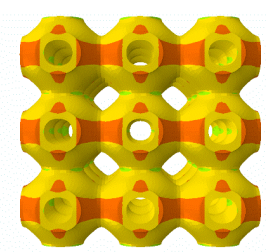

$\varepsilon_{y}=0.12$

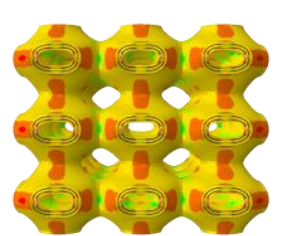

$\varepsilon_{y}=0.03$

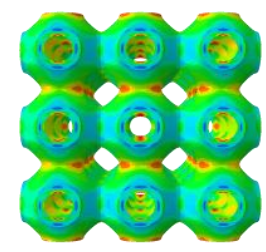

$\varepsilon_{y}=0.12$

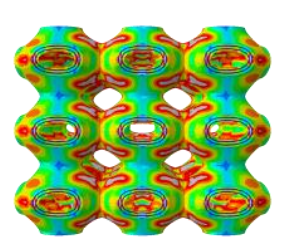

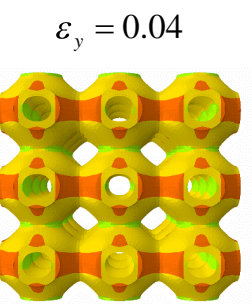

$\varepsilon_{y}=0.16$

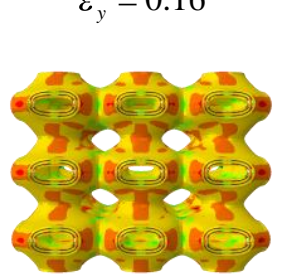

$\varepsilon_{y}=0.04$
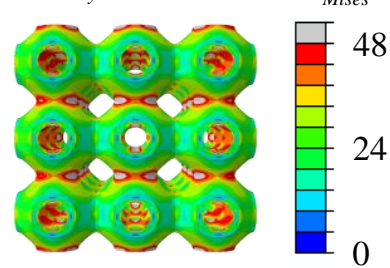

$\varepsilon_{y}=0.16$

$\sigma_{\text {Mises }}$

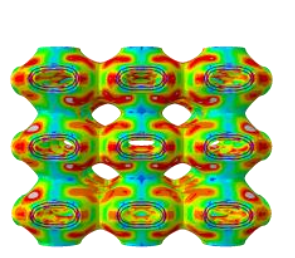

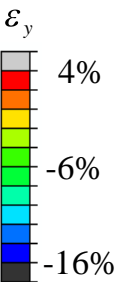

$\sigma_{\text {Mises }}$
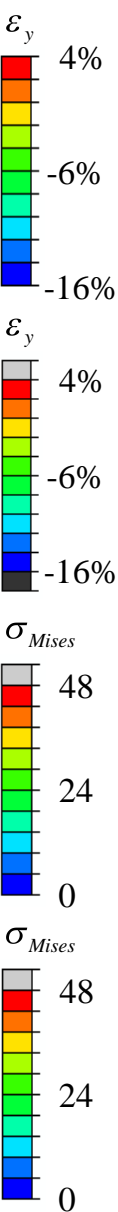

Figure S3. Simulated strain and von Mises stress evolution of the VW and MCC structures. (a)-

(b) Strain evolution of VW and MCC structures, respectively. (c)-(d) von Mises stress evolution of VW and MCC structures, respectively. The unit of von Mises stress is MPa.

\subsection{Cyclic loading-unloading tests}

We have discussed the deformation patterns of the first cycle for the compressive strain at $20 \%$ and $40 \%$ in our main text. Here, the deformation patterns of the remaining cycles for compressive strains of $20 \%$ and $40 \%$ are shown in Figures S4 and S5, respectively. For the compressive strain of $20 \%$, the sample already fractures when the compression begins at the $2^{\text {nd }}$ cycle (Figure S4 (a)). This explains the $23.8 \%$ decrease in stiffness compared with the $1^{\text {st }}$ cycle. With the strain increases, the crack propagates much deeper compared to the $1^{\text {st }}$ cycle. Most of 
the VeroWhitePlus phase is broken in the fractured zones, leaving soft phases connecting them. This decreases the maximum stress significantly. The $3^{\text {rd }}$ cycle is very much like the $2^{\text {nd }}$ one, except that some local areas like the top layer tend to buckle (Figure S4 (b)). This accounts for the almost negligible change of stiffness and maximum stress between the $2^{\text {nd }}$ and $3^{\text {rd }}$ cycles. For the $4^{\text {th }}$ cycle, some areas in the middle layer break with a consequent decrease of the maximum stress from the $3^{\text {rd }}$ to the $4^{\text {th }}$ cycles (Figure S4 (c)). During the $5^{\text {th }}$ cycle, some local surfaces tend to break so significantly that one part of the hole detaches, while another one also disconnects from it (Figure S4 (d)). It is interesting to notice that no new fracture paths are generated after the $1^{\text {st }}$ cycle. In other words, all the deformation after the $1^{\text {st }}$ cycle follows the path of that specific cycle. 

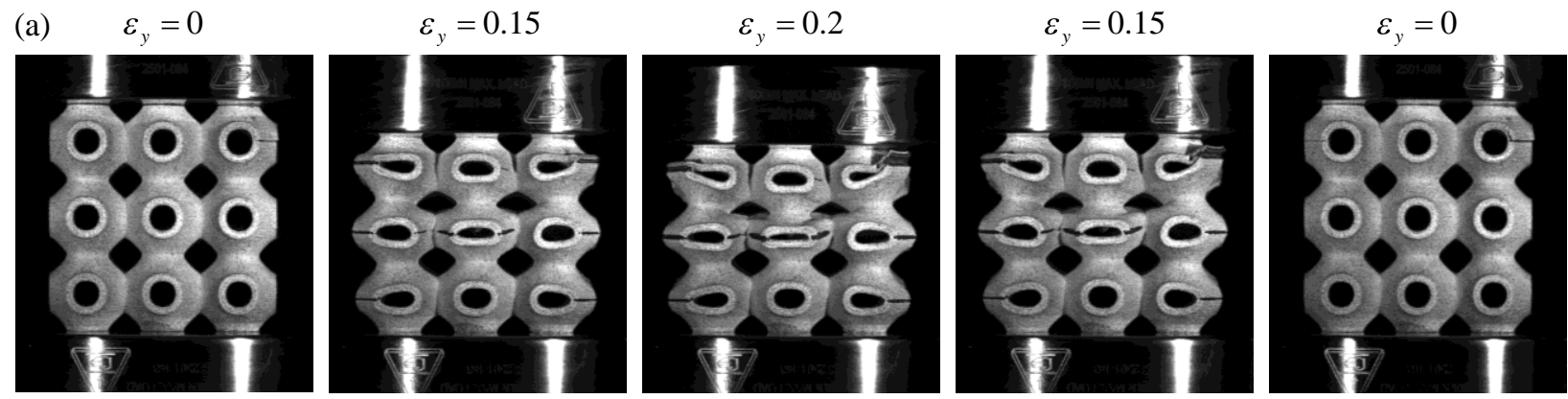

(b)
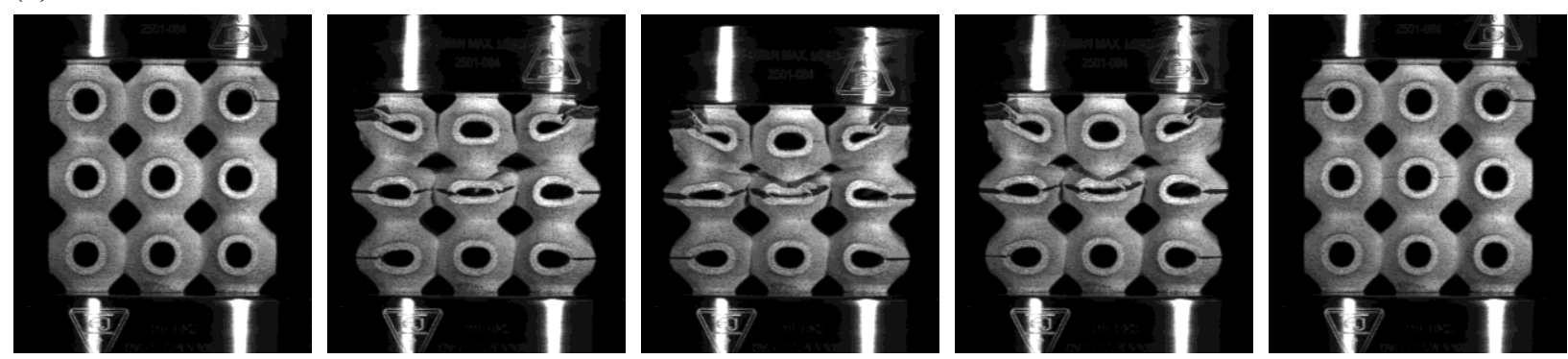

(c)
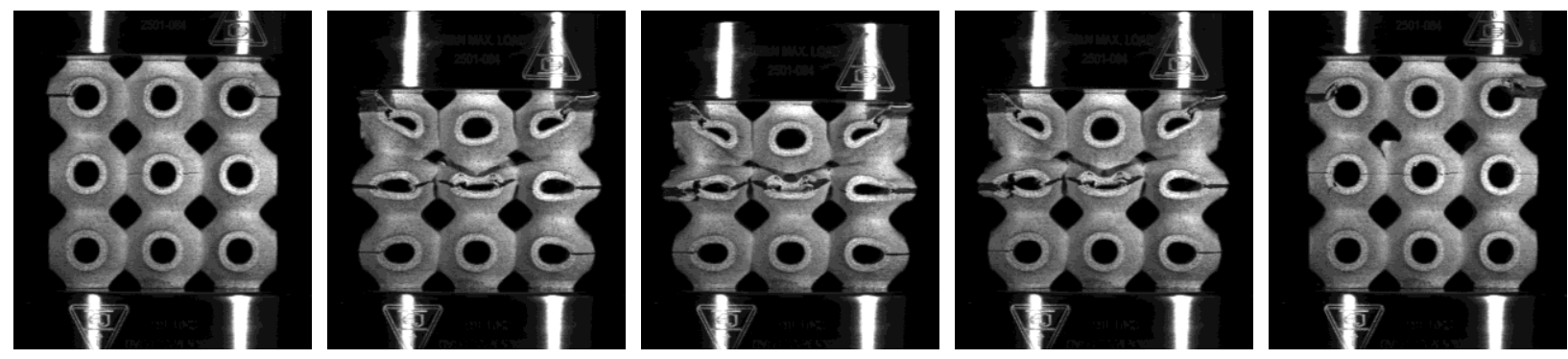

(d)
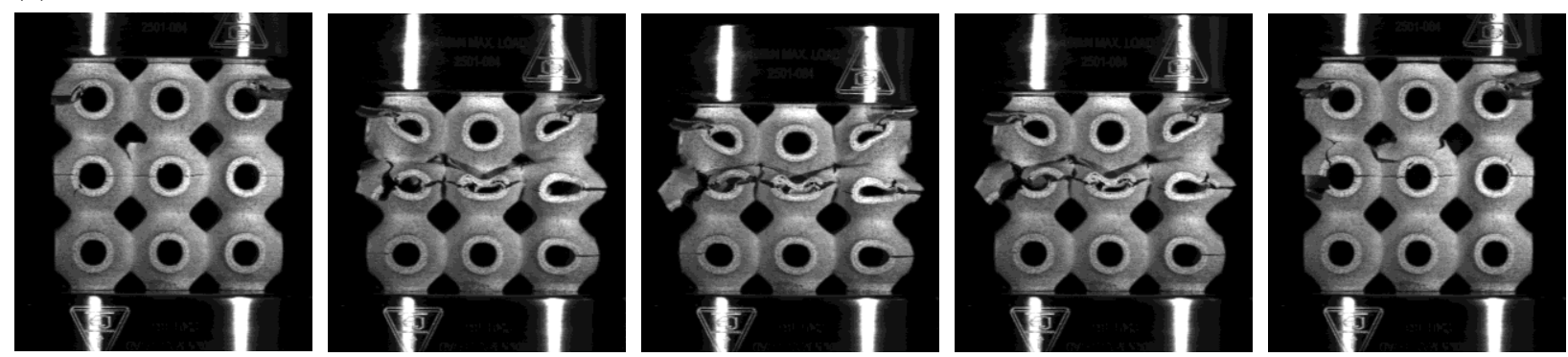

Figure S4. Deformation patterns at different strain levels when the MCC structure is compressed

up to $20 \%$. (a) 2nd cycle, (b) 3rd cycle, (c) 4th cycle, and (d) 5th cycle.

For a compressive strain of $40 \%$, one can observe the presence of cracks opening around the holes at the beginning of the $2^{\text {nd }}$ cycle (Figure S5 (a)). This justifies the $42.7 \%$ decrease in stiffness. Meanwhile, the first peak of stress also decreases significantly. This leads to the 
maximum stress drop of $35.4 \%$ and deferred from the first peak to the end. For the $3^{\text {rd }}$ cycle, the fractures almost penetrate the middle layer and some new fail zones are generated in the bottom layer at the beginning (Figure S5 (b)). This gives rise to the $67.9 \%$ decrease in stiffness, but not a significant change of the maximum stress. Note the presence of some small debris in the holes is produced by the dry paint used in the DIC experiments. During the $4^{\text {th }}$ cycle, the VeroWhitePlus of some local areas almost detaches from the sample (Figure S5 (c)). This accounts for the $97.1 \%$ decrease in stiffness compared to the $1^{\text {st }}$ cycle. During the $5^{\text {th }}$ cycle, almost all areas around the fractured surfaces are broken or weakly connected by small portions of the soft phase, explaining the $98.5 \%$ decrease in stiffness (Figure S5 (d)). 
(a)

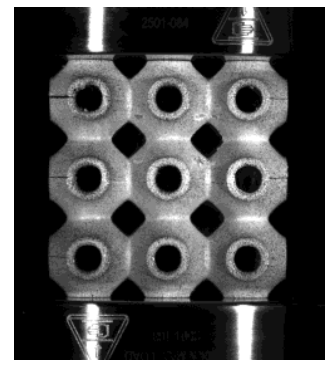

(b)

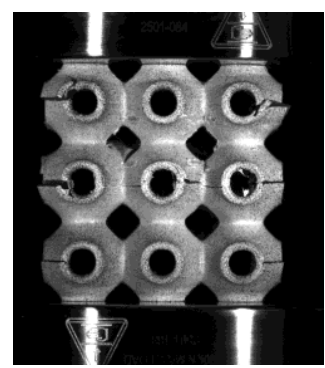

(c)
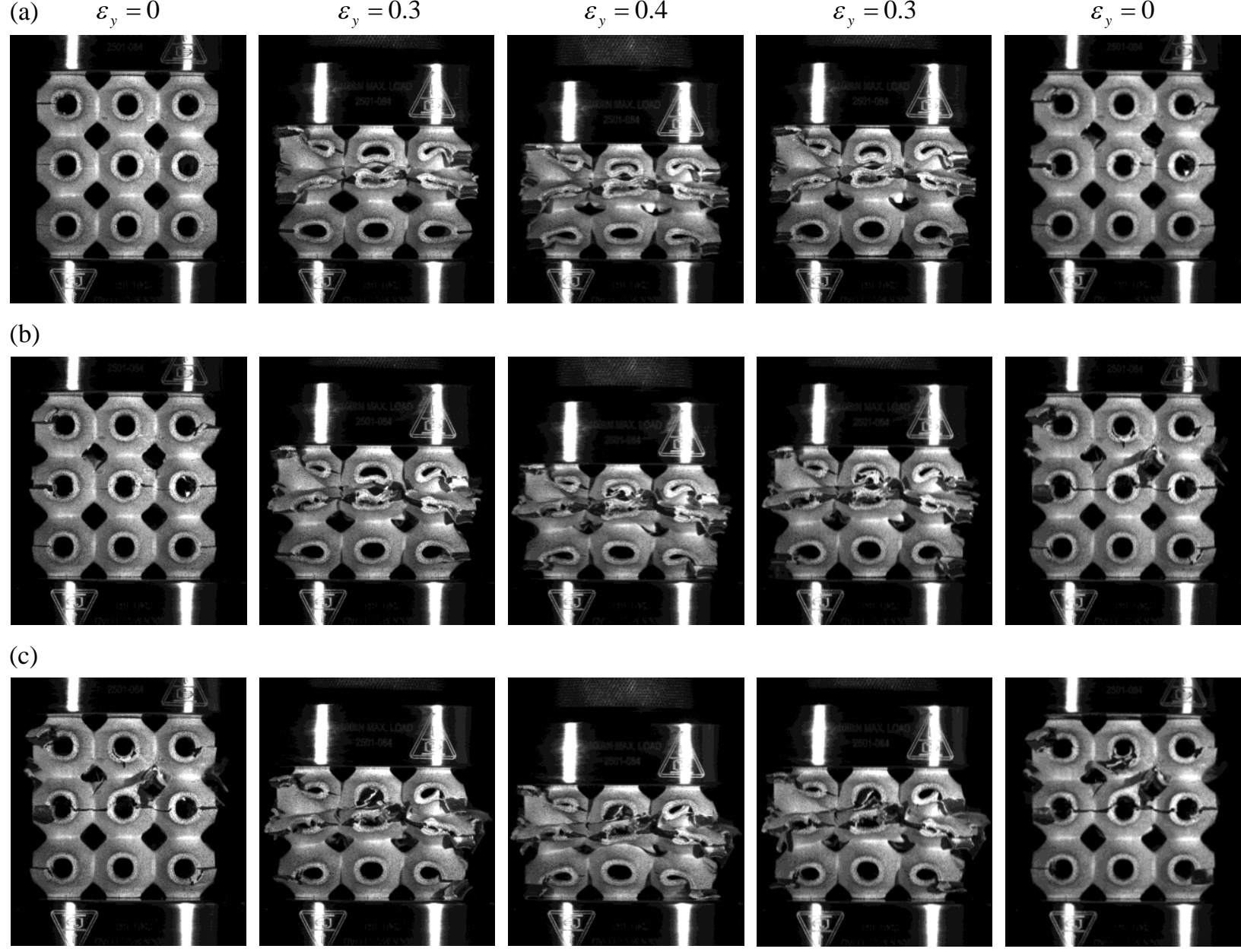

(d)
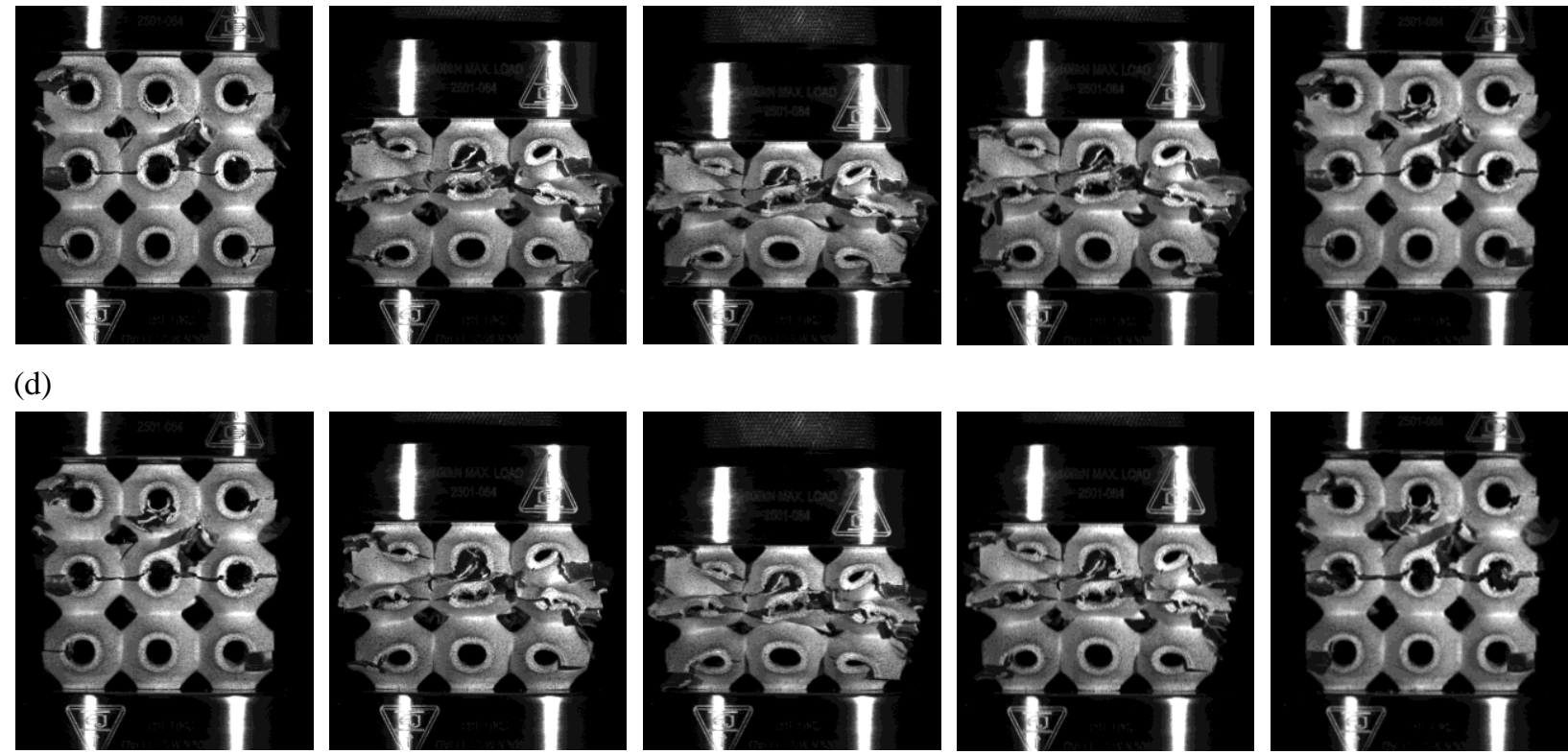

Figure S5. Deformation patterns at different strain levels when the MCC structure is compressed

up to $40 \%$. (a) 2nd cycle, (b) 3rd cycle, (c) 4th cycle, and (d) 5th cycle. 